\title{
Three-phase boundary motion by surface diffusion: stability of a mirror symmetric stationary solution
}

\author{
KAZUO ITO \\ Graduate School of Mathematics, Kyushu University, Ropponmatsu, Fukuoka 810-8560, Japan \\ AND \\ YOSHIHITO KOHSAKA \\ Department of Mathematics, Faculty of Science, Hokkaido University, Sapporo 060-0810, \\ Japan
}

[Received 2 September 1999 and in revised form 7 June 2000]

\begin{abstract}
We prove that the sharp interface model for a three-phase boundary motion by surface diffusion proposed by $\mathrm{H}$. Garcke and A. Novick-Cohen admits a unique global solution provided the initial data fulfils a certain symmetric criterion and is also close to a minimizer of the energy under an area constraint. This minimizer is also a stationary solution of the present model. Moreover, we prove that the global solution converges to the minimizer of the energy as time goes to infinity.
\end{abstract}

Keywords: Free boundary problem; triple junction; global existence; stability; surface diffusion

\section{Introduction and main results}

We study a sharp interface model for a three-phase boundary motion by surface diffusion proposed by H. Garcke and A. Novick-Cohen [15]. Let $\Omega \subset \mathbb{R}^{2}$ be a bounded domain. We consider a situation that three phases of a ternary alloy system in a non-equilibrium state are contained in $\Omega$ and they are separated by evolving three interphase boundaries $\Gamma^{i}(t), i=1,2,3$, where $t \geqslant 0$ denotes the time variable. These interfaces are connected by a triple junction $m(t) \in \Omega$ at their one end points and are perpendicular to the boundary of $\Omega, \partial \Omega$, at their other end points. It is known that a Cahn-Hilliard system with a concentration-dependent mobility describes this situation as a diffuse interface model. H. Garcke and A. Novick-Cohen [15] discussed a formal singular limit in this system to derive a sharp interface model for $\Gamma(t)=\bigcup_{i=1}^{3} \Gamma^{i}(t)$ with a triple junction $m(t)$ in the following: for $i=1,2,3$, and $t>0$

(A) along $\Gamma^{i}(t) ; \quad V^{i}=-l^{i} \sigma^{i} \kappa_{s s}^{i}$ : surface diffusion flow equation,

(B) at $\Gamma^{i}(t) \cap \partial \Omega$;

$\Gamma^{i}(t) \perp \partial \Omega$ : contact angle is $\pi / 2$,

$\kappa_{s}^{i}=0:$ no flux condition,

(C) at $m(t)$;

$\angle\left(\Gamma^{1}(t), \Gamma^{2}(t)\right)=\theta^{3}, \quad \angle\left(\Gamma^{2}(t), \Gamma^{3}(t)\right)=\theta^{1}, \quad \angle\left(\Gamma^{3}(t), \Gamma^{1}(t)\right)=\theta^{2}$,

$l^{1} \sigma^{1} \kappa_{s}^{1}=l^{2} \sigma^{2} \kappa_{s}^{2}=l^{3} \sigma^{3} \kappa_{s}^{3}$ : balance of fluxes,

$\sigma^{1} \kappa^{1}+\sigma^{2} \kappa^{2}+\sigma^{3} \kappa^{3}=0$ : continuity of the chemical potential 
with the initial condition

$$
\Gamma(0)=\Gamma_{0}=\bigcup_{i=1}^{3} \Gamma_{0}^{i}, \quad m(0)=m_{0} .
$$

Here, $V^{i}$ and $\kappa^{i}$ stand for the normal velocity and the curvature of $\Gamma^{i}(t)$ respectively, and $s$ denotes the arc-length parameter of $\Gamma^{i}(t)$. The sign convention used here is as follows: $s$ runs from $m(t)$, at which $s=0$, to the point of intersection of $\Gamma^{i}(t)$ with $\partial \Omega$, at which $s=L\left[\Gamma^{i}(t)\right]$, where $L\left[\Gamma^{i}(t)\right]$ denotes the total length of $\Gamma^{i}(t) ; V^{i}(t, s)$ and $\kappa^{i}(t, s)$ are computed to the direction of the unit normal $N^{i}(t, s)$ to $\Gamma^{i}(t)$ at $s$, where $\left(T^{i}(t, s), N^{i}(t, s)\right)$ makes the orthogonal coordinate system and $T^{i}(t, s)$ is the unit tangent to $\Gamma^{i}(t)$ at $s$. Moreover $l^{i}, \sigma^{i}$, and $\theta^{i}$ are positive constants with the constraints $\theta^{1}+\theta^{2}+\theta^{3}=2 \pi$ and

$$
\frac{\sigma^{1}}{\sin \theta^{1}}=\frac{\sigma^{2}}{\sin \theta^{2}}=\frac{\sigma^{3}}{\sin \theta^{3}}
$$

The latter condition is called Young's law which is well known among material scientists. $L\left(\Gamma^{i}(t), \Gamma^{j}(t)\right)$ stands for the angle between $\Gamma^{i}(t)$ and $\Gamma^{j}(t)$ for $i, j=1,2,3, i \neq j$. In [15] Garcke and Novick-Cohen also mathematically studied (1.1), (1.2) to obtain both a local existence result for $\Gamma_{0} \in C^{4+\alpha}(0<\alpha<1)$ with a suitable compatibility condition and a uniqueness result in a geometric sense.

Our purpose in this paper is to obtain a unique global solution $\Gamma(t)$ with a triple junction $m(t)$ of (1.1), (1.2) for $\Gamma_{0} \in C^{3}$ with a suitable compatibility condition in a symmetric framework and also to show its convergence to a stationary solution determined by the initial data as $t \rightarrow \infty$. As far as we know this is a first contribution to global results for the problem (1.1), (1.2).

Let us explain our framework for the problem (1.1), (1.2) which is devoted to study a symmetric evolution of $\Gamma(t)$ with respect to the $x$-axis. For this purpose let $\Omega=\left\{(x, y) \in \mathbb{R}^{2} ;-a<x<\right.$ $0,-b<y<b\}$, where $a$ and $b$ are positive constants large enough. We consider the evolution such that $\Gamma^{1}(t)$ always stays a segment on the $x$-axis, and $\Gamma^{2}(t)$ and $\Gamma^{3}(t)$ are symmetric with respect to the $x$-axis and $\Gamma^{3}(t)$ is in $\{(x, y) \in \Omega ; y \geqslant 0\}$. Let $\theta \in(0, \pi / 2)$ and set $\theta^{1}=2 \theta$ and $\theta^{2}=\theta^{3}=\pi-\theta$. For simplicity we put $\sigma^{2}=\sigma^{3}=1$ and $l^{1}=l^{2}=l^{3}=1$. Then Young's law and the condition on the balance of fluxes are simplified to $\sigma^{1}=2 \cos \theta$ and $\kappa_{s}^{1}=\kappa_{s}^{2}=\kappa_{s}^{3}=0$ at $m(t)$, respectively, and by the symmetry the condition on the continuity of the chemical potential is automatically fulfilled. Moreover, for brevity, we introduce two terminologies for union of three curves $\Gamma=\bigcup_{i=1}^{3} \Gamma^{i}$ with a triple junction $m$ that has the configuration as above. To do so, we set

$$
\mu[\xi]:=(-\xi, 0) \quad \text { for } \xi \in(0, a)
$$

and we then associate $\Gamma$ with a function $u:[-\xi, 0] \rightarrow[0, b)$ as follows:

$$
\Gamma=\Lambda[u, \xi], \quad m=\mu[\xi],
$$

where

$$
\begin{aligned}
& \Lambda[u, \xi]:=\bigcup_{i=1}^{3} \Lambda^{i}[u, \xi], \\
& \Lambda^{1}[u, \xi]:=\{(x, 0) ;-a \leqslant x \leqslant-\xi\}, \\
& \Lambda^{2}[u, \xi]:=\{(x,-u(x)) ;-\xi \leqslant x \leqslant 0\}, \\
& \Lambda^{3}[u, \xi]:=\{(x, u(x)) ;-\xi \leqslant x \leqslant 0\} .
\end{aligned}
$$


DEFINITION $1.1 \quad$ (i) Let $\theta \in(0, \pi / 2)$. We say that a union of three curves $\Gamma$ belongs to $\mathcal{S}_{\theta}$, if there are $\xi \in(0, a)$ and non-negative function $u \in C^{3}[-\xi, 0]$ with $u(-\xi)=0, u_{x}(-\xi)=$ $\tan \theta, u_{x}(0)=0$, and $\left(u_{x x}\left(1+u_{x}^{2}\right)^{-3 / 2}\right)_{x}=0$ at $x=-\xi$ and 0 such that $\Gamma=\Lambda[u, \xi]$ and $m=\mu[\xi]$.

(ii) Let $A>0$ be a given constant. We say that a union of three curves $\Gamma$ belongs to $\mathcal{C}_{A}$ if there are $\xi \in(0, a)$ and non-negative function $u \in C^{1}[-\xi, 0]$ with $u(-\xi)=0, u_{x}(0)=0$, and $\int_{-\xi}^{0} u(x) \mathrm{d} x=A$ such that $\Gamma=\Lambda[u, \xi]$ and $m=\mu[\xi]$.

Note that a union of three curves in $\mathcal{S}_{\theta}$ or in $\mathcal{C}_{A}$ is symmetric with respect to the $x$-axis. In view of the structure of the evolution problem (1.1), (1.2) it can be expected that if $\Gamma_{0} \in \mathcal{S}_{\theta}$, then the solution $\Gamma(t)$ of (1.1), (1.2) also belongs to $\mathcal{S}_{\theta}$ for all $t>0$ as long as it exists. So we proceed with the evolution problem (1.1), (1.2) on $\mathcal{S}_{\theta}$ and set $\Gamma(t)=\Lambda[u(t, \cdot), \xi(t)]$ with $m(t)=\mu[\xi(t)]$ for $t>0$ and $\Gamma_{0}=\Lambda\left[u_{0}, \xi_{0}\right]$ with $m_{0}=\mu\left[\xi_{0}\right]$. Then $(u(t, x), \xi(t))$ for $t \geqslant 0$ and $-\xi(t) \leqslant x \leqslant 0$ is an unknown function to be looked for and the equation (1.1) for $t>0$ is reduced to

$$
\left\{\begin{array}{l}
u_{t}=-\partial_{x}\left(\frac{1}{\left(1+u_{x}^{2}\right)^{1 / 2}} \partial_{x}\left(\frac{u_{x x}}{\left(1+u_{x}^{2}\right)^{3 / 2}}\right)\right), \quad-\xi(t)<x<0, \\
u_{x}(t,-\xi(t))=\tan \theta, \quad u_{x}(t, 0)=0, \\
\partial_{x}\left(\frac{u_{x x}}{\left(1+u_{x}^{2}\right)^{3 / 2}}\right)=0, \quad \text { at } x=-\xi(t) \text { and } 0, \\
u(t,-\xi(t))=0
\end{array}\right.
$$

and the initial condition (1.2) is reduced to

$$
u(0, x)=u_{0}(x) \quad \text { for }-\xi_{0}<x<0, \quad \xi(0)=\xi_{0} .
$$

Now our task is reduced to solving the problem (1.3), (1.4).

In the study of the global-in-time solvability of the evolution problem (1.3), (1.4), an energyminimizing result plays an essential role. To explain this, let $A>0$ satisfy $\xi_{\theta, A}<a$, where we put

$$
\begin{gathered}
\xi_{\theta, A}=r_{\theta, A} \sin \theta, \\
r_{\theta, A}=\left(\frac{2 A}{\theta-\sin \theta \cos \theta}\right)^{1 / 2} .
\end{gathered}
$$

(This choice of $A$ is only to ensure that $\Gamma_{\theta, A}$ defined below is completely contained in $\Omega$.) For such $A$, we also set

$$
u_{\theta, A}(x)=-r_{\theta, A} \cos \theta+\left(r_{\theta, A}^{2}-x^{2}\right)^{1 / 2}, \quad x \in\left[-\xi_{\theta, A}, 0\right]
$$

and define

$$
\Gamma_{\theta, A}=\Lambda\left[u_{\theta, A}, \xi_{\theta, A}\right]
$$


Then $\Gamma_{\theta, A}$ belongs to $\mathcal{C}_{A}$ and it consists of one segment $\Gamma_{\theta, A}^{1}=\Lambda^{1}\left[u_{\theta, A}, \xi_{\theta, A}\right]$ on the $x$-axis and two circular arcs $\Gamma_{\theta, A}^{i}=\Lambda^{i}\left[u_{\theta, A}, \xi_{\theta, A}\right], i=2,3$, which are symmetric to each other with respect to the $x$-axis. $\Gamma_{\theta, A}$ is also the unique stationary solution of (1.3) such that the area enclosed by $\Gamma_{\theta, A}^{3}$, the $x$-axis, and the $y$-axis is equal to $A$. Now we define the energy $E$ on $\mathcal{C}_{A}$ associated with the problem (1.3), (1.4) by

$$
E[\Gamma]=(a-\xi) \cos \theta+\int_{-\xi}^{0}\left(1+u_{x}^{2}\right)^{1 / 2} \mathrm{~d} x \quad \text { for } \Gamma=\Lambda[u, \xi] \in \mathcal{C}_{A} .
$$

Then it will be shown in Theorem 2.1 that $\Gamma_{\theta, A}$ is the unique minimizer of $E$ for $\Gamma=\Lambda[u, \xi] \in \mathcal{C}_{A}$. It is possible to say that this minimizing problem is an extended version of several kinds of isoperimetric problems with account of various boundary conditions, which were studied by many researchers by applying abstract methods such as the geometric measure theory. We especially refer the reader to [27]. But in Theorem 2.1 we shall give a direct proof, since our case can be treated by means of piecewise smooth graphs in one dimension. Owing to one-dimensionality, we have no extra difficulties to show the uniqueness and the existence of the minimizer of $E$ on $\mathcal{C}_{A}$, though some technical difficulties to be settled arise due to the presence of the triple junction.

The result in Theorem 2.1 is a key to investigating the global-in-time solvability of the problem (1.3), (1.4) that depends on the magnitude of $C^{2+\alpha}$-norm of the initial data with $0<\alpha \leqslant \frac{1}{2}$. In fact the $L^{2}$-estimate of the first derivative of the curvature of $\Gamma^{3}(t)$, which is the key estimate for the global solvability, needs the lower bound of $E[\Gamma(t)]$. The reason why the range of $\alpha$ is restricted to $\left(0, \frac{1}{2}\right]$ is, roughly speaking, due to the estimate of the $C^{\alpha}$-seminorm of $u_{x x}$ by the $L^{2}$-norm of $u_{x x x}$. Here we recall that Garcke and Novick-Cohen [15] previously presented the local existence theorem for (1.1), (1.2) for $\Gamma_{0}$ in $C^{4+\alpha}$ with $0<\alpha<1$. So when we try to construct a global solution by extending a local solution, we are forced to improve their result to reconstruct a local solution for $\Gamma_{0}$ having at most $C^{2+\alpha}$-regularity with $0<\alpha \leqslant \frac{1}{2}$. This issue can be settled by applying the optimal regularity result for parabolic problems, as comprehensively studied in [21].

Next we should mention that there are two remarkable features of the evolution problem (1.1), (1.2); one is the area-preserving property and another is the energy-decreasing property [15]. The former means that each area of the ' $\Delta$ '-shaped domains enclosed by $\Gamma^{1}(t) \cup \Gamma^{2}(t) \cup \partial \Omega$ and by $\Gamma^{2}(t) \cup \Gamma^{3}(t) \cup \partial \Omega$ is preserved for all $t \geqslant 0$ under the motion by (1.1). The latter means that the energy associated with (1.1) for the solution $\Gamma(t)$ of (1.1) is a non-increasing function of $t$. It will be shown in Section 4 that these two properties as well as the results in Section 2 enable us to obtain an a priori estimate of the first derivative of the curvature of $\Gamma^{3}(t)$ in $L^{2}$ when the initial curve $\Gamma_{0}$ and $\Gamma_{\theta}, A_{0}$ are close to each other in some sense, where $A_{0}$ denotes the area enclosed by $\Gamma_{0}^{3}$, the $x$-axis, and the $y$-axis. This procedure originates from $\mathrm{X}$. Chen [5] and C. M. Elliott and H. Garcke [8] where the motions of closed curves are treated. In our case the motions of the triple junction and the end points of $\Gamma(t)$ at $\partial \Omega$ must be also taken into account. When $\theta \in(0, \pi / 2)$, we can settle this issue to establish a modified version of their estimates.

Thus, one can expect that if $\Gamma_{0}$ is close to $\Gamma_{\theta}, A_{0}$, then a global solution $\Gamma(t)$ with a triple junction $m(t)$ of (1.3), (1.4) exists and converges to $\Gamma_{\theta, A_{0}}$ as $t \rightarrow \infty$. This is in fact the case and we shall show it in our main theorem. To do so it is convenient to introduce the following notations. 
Notation. Let $I=[0,1]$. For $0 \leqslant t_{0}<t_{1}<\infty$ we set $R_{t_{0}, t_{1}}=\left(t_{0}, t_{1}\right] \times I$. For $0<\alpha<1$ we define spaces of functions $v(t, \eta)$ for $(t, \eta) \in\left[t_{0}, t_{1}\right] \times I$ and $\xi(t)$ for $t \in\left[t_{0}, t_{1}\right]$ as

$$
\begin{aligned}
& \mathcal{Y}^{2+\alpha}\left(\overline{R_{t_{0}, t_{1}}}\right)=C^{0,2+\alpha}\left(\overline{R_{t_{0}, t_{1}}}\right) \cap C^{1 / 2}\left(\left[t_{0}, t_{1}\right] ; C^{\alpha}(I)\right), \\
& \mathcal{Y}^{4+\alpha}\left(\overline{R_{t_{0}, t_{1}}}\right)=\left\{v \in \mathcal{Y}^{2+\alpha}\left(\overline{R_{t_{0}, t_{1}}}\right) \cap C^{1,4+\alpha}\left(R_{t_{0}, t_{1}}\right) ;\|v\|_{\mathcal{Y}^{4+\alpha}\left(\overline{R_{t_{0}, t_{1}}}\right)}<\infty\right\}, \\
& \mathcal{Z}^{1}\left[t_{0}, t_{1}\right]=\left\{\xi \in C\left[t_{0}, t_{1}\right] \cap C^{1}\left(t_{0}, t_{1}\right] ;\|\xi\|_{\mathcal{Z}^{1}\left[t_{0}, t_{1}\right]}<\infty\right\},
\end{aligned}
$$

where the norms with which the latter two spaces are equipped are defined by

$$
\begin{aligned}
\|v\|_{\mathcal{Y}^{4+\alpha}\left(\overline{R_{t_{0}, t_{1}}}\right)}= & \|v\|_{\mathcal{Y}^{2+\alpha}\left(\overline{R_{t_{0}, t_{1}}}\right)}+\sup _{0<\delta<t_{1}-t_{0}} \delta^{1 / 4}\left\|v_{\eta \eta \eta}\right\|_{C^{0, \alpha}\left(\overline{R_{t_{0}+\delta, t_{1}}}\right)} \\
& +\sup _{0<\delta<t_{1}-t_{0}} \delta^{1 / 2}\left\|v_{\eta \eta \eta \eta}\right\|_{C^{0, \alpha}\left(\overline{R_{t_{0}}+\delta, t_{1}}\right)}+\sup _{0<\delta<t_{1}-t_{0}} \delta^{1 / 2}\left\|v_{t}\right\|_{C^{0, \alpha}\left(\overline{R_{t_{0}+\delta, t_{1}}}\right)}, \\
\|\xi\|_{\mathcal{Z}^{1}\left[t_{0}, t_{1}\right]}= & \|\xi\|_{C\left[t_{0}, t_{1}\right]}+\sup _{0<\delta<t_{1}-t_{0}} \delta^{1 / 2}\|\dot{\xi}\|_{C\left[t_{0}+\delta, t_{1}\right]} .
\end{aligned}
$$

Here the spaces such as $C^{j, k+\beta}\left(\left[t_{0}, t_{1}\right] \times I\right)(0 \leqslant j \leqslant(k+\beta) / 4, k=0,1, \ldots, 0<\beta<1)$ used here are defined in the usual manner; for their complete descriptions, see [22] or [21].

The manner of the establishment of the above spaces is somewhat complicated but it will be seen in Section 3 that they are quite reasonable when they are devoted to the study of the local-in-time solvability for (1.3), (1.4) with $\Gamma_{0} \in \mathcal{S}_{\theta}$. We also remind the reader that the indices of $\mathcal{Y}$-spaces measure the space regularities, whereas the index of $\mathcal{Z}$-space measures the time regularity.

Now we are in position to state our main result.

Theorem 1.2 Let $\theta \in(0, \pi / 2)$ and let $\Gamma_{0}=\Lambda\left[u_{0}, \xi_{0}\right] \in \mathcal{S}_{\theta}$. Assume that $\Gamma_{0}$ and $\Gamma_{\theta, A_{0}}$ are close to each other in the sense that

$$
\left\|\kappa_{0, s}^{3}\right\|_{L^{2}\left(\Gamma_{0}^{3}\right)}^{2}+C_{\theta, A_{0}}\left(E\left[\Gamma_{0}\right]-E\left[\Gamma_{\theta, A_{0}}\right]\right) \text { is sufficiently small, }
$$

here $\kappa_{0}^{3}(s)$ is the curvature of $\Gamma_{0}^{3}$ with the argument of its arc-length parameter $s$ and $C_{\theta, A_{0}}$ is a positive constant depending on $\theta$ and $A_{0}$. Then,

(i) the problem (1.3), (1.4) admits a unique global solution $\Gamma(t)=\Lambda[u(t, \cdot), \xi(t)]$ with a triple junction $m(t)=\mu[\xi(t)]$ for $t \geqslant 0$ satisfying

$$
(v, \xi) \in \mathcal{Y}^{4+\alpha}\left(\overline{R_{0, T}}\right) \times \mathcal{Z}^{1}[0, T] \quad \text { for any } T>0
$$

with $\alpha \in(0,1 / 2]$, where

$$
v(t, \eta):=u(t,-(1-\eta) \xi(t)), \quad(t, \eta) \in[0, \infty) \times I .
$$

(ii) Moreover the solution $\Gamma(t)$ converges to $\Gamma_{\theta, A_{0}}$ uniformly and $E[\Gamma(t)]$ also converges to $E\left[\Gamma_{\theta, A_{0}}\right]$ as $t \rightarrow \infty$.

Note that $E\left[\Gamma_{0}\right]-E\left[\Gamma_{\theta, A_{0}}\right]$ is positive, since $\Gamma_{\theta, A_{0}}$ is the unique minimizer of $E$ on $\mathcal{C}_{A_{0}}$ as mentioned above. 
REMARK 1.3 (i) The equation which describes evolving interface moved by surface diffusion was first proposed by W. W. Mullins [24] and it takes the form

$$
V=-\Delta_{\Gamma(t)} H \quad \text { on } \Gamma(t) \text { for } t>0,
$$

where $\Gamma(t)$ is an unknown hypersurface with no boundary; $V, H$, and $\Delta_{\Gamma(t)}$ stand for the outward normal velocity, the outward mean curvature, and the Laplace-Beltrami operator of $\Gamma(t)$, respectively. We refer the reader to $[3,4,7]$ for recent developments concerning the derivation of (1.11) and also to $[6,8,10,11,16,17,20]$ and references cited therein for both analytic and numerical results. For (1.11) it is known that a loss of embeddedness ( [16] for curves, [23] for surfaces) and a graph-breaking [9] for $\Gamma(t)$ may occur for a class of the initial data. Hence (1.10) seems necessary.

(ii) For closed curves there are several results related to Theorem 1.2. X. Chen [5] showed for the Hele-Shaw problem that if the initial closed curve $\Gamma_{0}$ is close to a circle, then the global solution $\Gamma(t)$ of the Hele-Shaw problem exists and converges to a (possibly another) circle with the same area enclosed by $\Gamma_{0}$ as $t \rightarrow \infty$. Elliott and Garcke [8] also obtained the same results for other area-preserving and curve-shortening motions including (1.11). Escher et al. [10-13] extended their results to higher-dimensional versions by means of both Amann's sophisticated theory for analytic semigroups and a centre manifold analysis. For further information, see the references cited in [12].

(iii) For the second-order case there are several results related to the present problem. D. Hilhorst and J. Hulshof [19] showed for the heat equation with similar angle condition to our problem that the solution $\Gamma(t)$ exists in $[0, T]$ for a finite time $T>0$ and is asymptotically equal to a self-similar shrinking solution which vanishes at $T$. Galaktionov et al. [14] extended the result of [19] to the radially symmetric multi-dimensional case. For the three-phase problem with a triple junction, L. Bronsard and F. Reitich [2] dealt with the mean curvature evolution for phase boundaries which are coupled by an angle condition, known as Young's law, at a triple junction. They showed the local-time existence of that problem.

This paper proceeds as follows. In Section 2 we prove that the unique minimizer of $E$ on $\mathcal{C}_{A}$ is $\Gamma_{\theta, A}$. Next, by means of an optimal regularity result for parabolic problems (as studied in [21]), in Section 3 we show a local existence result of (1.3), (1.4) depending on the magnitude of $\Gamma_{0} \in C^{2+\alpha}$ $(0<\alpha<1)$, Theorem 3.1, which is an improvement of the result obtained in [15] since Garcke and Novick-Cohen [15] treated $C^{4+\alpha}$-initial data. This improvement is necessary to obtain global solutions $\Gamma(t)$ of (1.3), (1.4) by means of a priori estimates of solutions in $C^{2+\alpha}$ with $0<\alpha \leqslant \frac{1}{2}$. For the reader's convenience we show an essential part of calculations employed in the proof of Theorem 3.1 in the Appendix. By virtue of the results in Sections 2 and 3 and the assumption (1.10) as well as both area-preserving and energy-decreasing properties, in Section 4 we obtain an a priori estimate of the solution $\Gamma(t)$ in $C^{2+\alpha}\left(0<\alpha \leqslant \frac{1}{2}\right)$ for $t \geqslant 0$ and consequently get a unique global solution for (1.3), (1.4), which proves Theorem 1.2(i). Finally, in Section 5, we prove Theorem 1.2(ii) in Theorems 5.5 and 5.6.

\section{Unique minimizer of the energy}

This section is devoted to investigating the stationary problem associated with the non-stationary one (1.3), (1.4). This problem is the energy-minimizing problem under an area-constraint in the 
following:

$$
\text { minimize } E \text { on } \mathcal{C}_{A} \text {, }
$$

where $\mathcal{C}_{A}$ is as in Definition 1.1(ii).

The result is stated as follows.

TheOREM 2.1 (Unique existence of the minimizer). Let $A>0$. Then the functional $E: \mathcal{C}_{A} \rightarrow \mathbb{R}$ has a unique minimizer in $\mathcal{C}_{A}$, which coincides with $\Gamma_{\theta, A}$ in (1.8).

Proof. We shall prove this result by using a kind of isoperimetric inequality under the constraint that the triple junction $m=(-\xi, 0)$ of $\Gamma \in \mathcal{C}_{A}$ is fixed arbitrarily. This isoperimetric inequality gives an information on the lower bound of the length of $\Gamma^{3}$, which is attained at the length of a circular arc having one end point $(-\xi, 0)$ (more precise descriptions are given in Step (a) below). Thus our preliminary task is to investigate this length of the circular arc when $\xi$ moves from $-a$ to 0 . The proof proceeds via four steps.

(a) The fundamental tool to prove Theorem 2.1 is a kind of isoperimetric inequality described below.

LEMMA 2.2 Let $\xi$ and $A$ be positive constants and fixed. Let $\Sigma$ be any $C^{1}$-simple curve in $\{(x, y) ; x \leqslant 0, y \geqslant 0\}$ satisfying the following (i)-(iii):

(i) one end point is $(-\xi, 0)$ and another end point is on the positive $y$-axis,

(ii) $\Sigma$ intersects the $y$-axis perpendicularly,

(iii) the area enclosed by $\Sigma$, the $x$-axis, and the $y$-axis is equal to $A$. Moreover let $\Sigma_{\xi, A}$ be the unique circular arc in $\{(x, y) ; x \leqslant 0, y \geqslant 0\}$ satisfying the above conditions (i)-(iii). Then it holds that

$$
L[\Sigma] \geqslant L\left[\Sigma_{\xi, A}\right]
$$

where $L[\cdot]$ denotes the length of the curve. The equality sign holds only when $\Sigma=\Sigma_{\xi, A}$.

The proof of Lemma 2.2 can be obtained by a slight modification of that of the standard isoperimetric inequality for simple closed curves, so we omit this proof.

(b) Let $\Sigma_{\xi, A}$ be as above and we then investigate $\rho(\xi):=L\left[\Sigma_{\xi, A}\right]$ for $\xi>0$ as an independent variable and with $A>0$ still fixed.

We denote by $\psi \in(0, \pi)$ the angle of $\Sigma_{\xi, A}$ from the $x$-axis at $(-\xi, 0)$. Then an elementary geometric observation shows that

$$
\rho(\xi)=\xi \frac{\psi}{\sin \psi}
$$

On the other hand, by an elementary calculation based on the area constraint, one can show that $\psi$ and $\xi$ must satisfy the relation

$$
\frac{2 A}{\xi^{2}}=\frac{\psi-\sin \psi \cos \psi}{\sin ^{2} \psi} \quad \text { for } \xi>0 .
$$


We denote by $S(\psi)$ the right-hand side of (2.4). It is straightforward to check that $S:(0, \pi) \rightarrow \mathbb{R}$ is monotone increasing. Hence we can solve (2.4) with respect to $\psi$ as

$$
\psi=\Psi(\xi):=S^{-1}\left(\frac{2 A}{\xi^{2}}\right)
$$

Thus we obtain from (2.3) and (2.5) that $\rho(\xi)=\xi \frac{\Psi(\xi)}{\sin \Psi(\xi)}$ for $\xi>0$. Differentiating $\rho(\xi)$ and using (2.4), we can easily derive the formula

$$
\rho^{\prime}(\xi)=\cos \Psi(\xi)
$$

(c) Now we investigate $E[\Gamma]$ for $\Gamma \in \mathcal{C}_{A}$ with the triple junction $m=(-\xi, 0)$. Lemma 2.2 implies

$$
\begin{aligned}
E[\Gamma] & =(a-\xi) \cos \theta+L\left[\Gamma^{3}\right] \\
& \geqslant(a-\xi) \cos \theta+\rho(\xi)=: F(\xi) .
\end{aligned}
$$

We investigate the lower bound of $F(\xi)$. Using (2.6), we have

$$
\begin{aligned}
F^{\prime}(\xi) & =\cos \Psi(\xi)-\cos \theta \\
& =\cos \Psi(\xi)-\cos \Psi\left(\xi_{\theta, A}\right),
\end{aligned}
$$

where $\xi_{\theta, A}$ is in (1.5). Since the function $\xi \mapsto \cos \Psi(\xi)$ is monotone increasing, we conclude that $F(\xi)$ takes its unique minimum only at $\xi=\xi_{\theta, A}$ and then $F\left(\xi_{\theta, A}\right)=E\left[\Gamma_{\theta, A}\right]$.

(d) Thus we arrive at

$$
E[\Gamma] \geqslant(a-\xi) \cos \theta+\rho(\xi) \geqslant E\left[\Gamma_{\theta, A}\right] \quad \text { for } \Gamma \in \mathcal{C}_{A}
$$

This shows that $\Gamma_{\theta, A}$ is a minimizer of $E$ on $\mathcal{C}_{A}$.

We shall show that $\Gamma_{\theta, A}$ is the unique minimizer of $E$ on $\mathcal{C}_{A}$. In order to show this, we assume that $E[\Gamma]=E\left[\Gamma_{\theta}, A\right]$ for a $\Gamma \in \mathcal{C}_{A}$, and we then imply $\Gamma=\Gamma_{\theta, A}$. Under the above assumption, both two inequality signs in (2.7) must be equality signs. It follows from Lemma 2.2 that for each $\xi>0$, the first equality sign holds only when $\Gamma^{3}=\Sigma_{\xi, A}$. In addition, by (c) the second equality sign holds only when $\xi=\xi_{\theta, A}$. Hence we conclude that $\Gamma$ must coincide with $\Gamma_{\theta, A}$. This completes the proof.

We can also obtain a lower bound of $L\left[\Gamma^{3}\right]$ for $\Gamma \in \mathcal{C}_{A}$.

LEMMA 2.3 It holds for $\Gamma \in \mathcal{C}_{A}$

$$
L\left[\Gamma^{3}\right] \geqslant \sqrt{\pi A} .
$$

Proof. Let $m=(-\xi, 0)$ be the triple junction of $\Gamma$. Then, as stated in (c) in the proof of Theorem 2.1, it holds that $L\left[\Gamma^{3}\right] \geqslant \rho(\xi)$. By (2.6) we see that $\rho$ takes its unique minimum at $\xi_{*}$ satisfying $\Psi\left(\xi_{*}\right)=\pi / 2$. It then follows from (2.4) that $\xi_{*}=2 \sqrt{A / \pi}$. Thus we have $\rho\left(\xi_{*}\right)=\xi_{*} \Psi\left(\xi_{*}\right) / \sin \Psi\left(\xi_{*}\right)=\sqrt{\pi A}$. This completes the proof. 


\section{Local existence}

In this section, we study a local existence result for (1.3), (1.4) for the initial data $\Gamma_{0} \in C^{2+\alpha}$ with $0<\alpha<1$. We once again point out that $\mathrm{H}$. Garcke and A. Novick-Cohen [15] previously obtained a local existence result for (1.1), (1.2) when the initial data $\Gamma_{0}$ belongs to $C^{4+\alpha}$ with $0<\alpha<1$. Our result is an improvement of that of [15] in the sense that the existence time of the local solution depends upon the magnitude of $\Gamma_{0}$ in $C^{2+\alpha}$ with $0<\alpha<1$. This improvement is useful in investigating the global solvability for (1.3), (1.4), which will be clarified in Section 4. In order to obtain our local existence results for the less regular data, we shall use the optimal regularity theory for parabolic problems as in [21] instead of the theory by V. A. Solonnikov [25] used in [15].

We shall first derive the equation for $\xi$. To do so we assume that $(u, \xi)$ is a solution of the problem (1.3) without the equation $u(t,-\xi(t))=0$ and (1.4) under the condition $u_{0}\left(-\xi_{0}\right)=0$. Then the equation $u(t,-\xi(t))=0$ and

$$
\dot{\xi}(t)=-C_{1}(\theta) u_{x x x x}(t,-\xi(t))+C_{2}(\theta) u_{x x}^{3}(t,-\xi(t))
$$

are equivalent, where $C_{1}(\theta):=1 /\left[\left(1+\tan ^{2} \theta\right)^{2} \tan \theta\right], C_{2}(\theta):=3\left(1+5 \tan ^{2} \theta\right) /\left[\left(1+\tan ^{2} \theta\right)^{4} \tan \theta\right]$. Here and hereafter, for simplicity of the notation we often use $\dot{\xi}$ instead of $\mathrm{d} \xi / \mathrm{d} t$. In order to see this equivalence, we first assume that the equation $u(t,-\xi(t))=0$ is satisfied. We differentiate it with respect to $t$ and use the boundary conditions $u_{x}(t,-\xi(t))=\tan \theta$, and $\partial_{x}\left(u_{x x} /\left(1+u_{x}^{2}\right)^{3 / 2}\right)=0$ at $x=-\xi(t)$. Then we get (3.1). Conversely, if (3.1) is satisfied, then we integrate it with respect to $t$. By virtue of the condition $u_{0}\left(-\xi_{0}\right)=0$, we then get the equation $u(t,-\xi(t))=0$.

From now on, we shall study the problem (1.3) without the equation $u(t,-\xi(t))=0,(1.4)$ and (3.1) with $\xi(0)=\xi_{0}$ for the initial data $\left(u_{0}(x), \xi_{0}\right)$ satisfying $u_{0}\left(-\xi_{0}\right)=0, u_{0 x}\left(-\xi_{0}\right)=\tan \theta$ and $u_{0 x}(0)=0$.

In order to normalize the coordinate $x$, we perform the change of variables for each $t \geqslant 0$ as follows:

$$
\eta=1+\frac{x}{\xi(t)}, \quad v(t, \eta)=u(t,-(1-\eta) \xi(t)) .
$$

Then, the problem (1.3) without the equation $u(t,-\xi(t))=0,(1.4)$ and (3.1) become the form for $(t, \eta) \in(0, T) \times(0,1)$ :

$$
\begin{aligned}
& v_{t}=f\left(\eta, v_{\eta}, v_{\eta \eta}, v_{\eta \eta \eta}, v_{\eta \eta \eta \eta}, \xi, \dot{\xi}\right), \\
& v_{\eta}(t, 0)=\xi(t) \tan \theta, \\
& v_{\eta}(t, 1)=0, \\
& v_{\eta \eta \eta}(t, 0)=\frac{3 \tan \theta}{1+\tan ^{2} \theta} \cdot \frac{v_{\eta \eta}^{2}(t, 0)}{\xi(t)}, \\
& v_{\eta \eta \eta}(t, 1)=0, \\
& v(0, \eta)=v_{0}(\eta):=u_{0}\left(-(1-\eta) \xi_{0}\right), \\
& \dot{\xi}(t)=-C_{1}(\theta) \frac{v_{\eta \eta \eta \eta}(t, 0)}{\xi^{4}(t)}+C_{2}(\theta) \frac{v_{\eta \eta}^{3}(t, 0)}{\xi^{6}(t)}, \\
& \xi(0)=\xi_{0},
\end{aligned}
$$


where

$$
\begin{aligned}
& f\left(\eta, v_{\eta}, v_{\eta \eta}, v_{\eta \eta \eta}, v_{\eta \eta \eta \eta}, \xi, \dot{\xi}\right) \\
& \quad=-\frac{1}{\left(\xi^{2}+v_{\eta}^{2}\right)^{2}} v_{\eta \eta \eta \eta}+\frac{10 v_{\eta} v_{\eta \eta}}{\left(\xi^{2}+v_{\eta}^{2}\right)^{3}} v_{\eta \eta \eta}+\frac{3\left(\xi^{2}-5 v_{\eta}^{2}\right) v_{\eta \eta}^{3}}{\left(\xi^{2}+v_{\eta}^{2}\right)^{4}}-\frac{(1-\eta) \dot{\xi} v_{\eta}}{\xi} .
\end{aligned}
$$

Thus our task is reduced to solve the problem (3.2)-(3.9) locally in time.

THEOREM 3.1 (Local existence) Let $\alpha \in(0,1)$. Let us assume that $v_{0} \in C^{2+\alpha}(I)$, and that $v_{0}$ satisfies $v_{0}(0)=0, v_{0 \eta}(0)=\xi_{0} \tan \theta, v_{0 \eta}(1)=0$. Let us also assume that $\xi_{0}>0$. Then, there exists a $T_{1}=T_{1}\left(\xi_{0}, 1 /\left\|v_{0}\right\|_{C^{2+\alpha}(I)}\right)>0$ such that the problem (3.2)-(3.9) has a unique solution $(v, \xi) \in \mathcal{Y}^{4+\alpha}\left(\overline{R_{0, T_{1}}}\right) \times \mathcal{Z}^{1}\left[0, T_{1}\right]$.

Before proving this theorem, we need some preliminaries. We shall first linearize (3.2), (3.9) about the initial data. For later convenience, we introduce a parameter $\tau \geqslant 0$, which is regarded as initial time. If a pair of functions $(\bar{v}, \bar{\xi})$, which has a suitable regularity, is given, then, for $C^{4}$ function $U:[0,1] \ni \eta \rightarrow U(\eta) \in \mathbb{R}$, we define a differential operator $\mathcal{A}_{\tau}$ by

$$
\begin{aligned}
\mathcal{A}_{\tau} U:= & -\frac{1}{\left(\bar{\xi}^{2}(\tau)+\bar{v}_{\eta}^{2}(\tau, \eta)\right)^{2}} \partial_{\eta}^{4} U+10 \frac{\bar{v}_{\eta}(\tau, \eta) \bar{v}_{\eta \eta}(\tau, \eta)}{\left(\bar{\xi}^{2}(\tau)+\bar{v}_{\eta}^{2}(\tau, \eta)\right)^{3}} \partial_{\eta}^{3} U \\
& +C_{1}(\theta)(1-\eta) \frac{\bar{v}_{\eta}(\tau, \eta)}{\bar{\xi}^{5}(\tau)} \partial_{\eta}^{4} U(0)
\end{aligned}
$$

In addition, we set

$$
\begin{aligned}
F_{\tau}(t, \eta):= & -\left\{\frac{1}{\left(\bar{\xi}^{2}(t)+\bar{v}_{\eta}^{2}(t, \eta)\right)^{2}}-\frac{1}{\left(\bar{\xi}^{2}(\tau)+\bar{v}_{\eta}^{2}(\tau, \eta)\right)^{2}}\right\} \bar{v}_{\eta \eta \eta \eta}(t, \eta) \\
& +10\left\{\frac{\bar{v}_{\eta}(t, \eta) \bar{v}_{\eta \eta}(t, \eta)}{\left(\bar{\xi}^{2}(t)+\bar{v}_{\eta}^{2}(t, \eta)\right)^{3}}-\frac{\bar{v}_{\eta}(\tau, \eta) \bar{v}_{\eta \eta}(\tau, \eta)}{\left(\bar{\xi}^{2}(\tau)+\bar{v}_{\eta}^{2}(\tau, \eta)\right)^{3}}\right\} \bar{v}_{\eta \eta \eta}(t, \eta) \\
& +C_{1}(\theta)(1-\eta)\left(\frac{\bar{v}_{\eta}(t, \eta)}{\bar{\xi}^{5}(t)}-\frac{\bar{v}_{\eta}(\tau, \eta)}{\bar{\xi}^{5}(\tau)}\right) \bar{v}_{\eta \eta \eta \eta}(t, 0) \\
& +\frac{3\left(\bar{\xi}^{2}(t)-5 \bar{v}_{\eta}^{2}(t, \eta)\right) \bar{v}_{\eta \eta}^{3}(t, \eta)}{\left(\bar{\xi}^{2}(t)+\bar{v}_{\eta}^{2}(t, \eta)\right)^{4}}-C_{2}(\theta) \frac{(1-\eta) \bar{v}_{\eta}(t, \eta)}{\bar{\xi}^{7}(t)} \bar{v}_{\eta \eta}^{3}(t, 0) .
\end{aligned}
$$

In the proof of Theorem 3.1, we use $\tau=0$. Let $T>0$. Then, for given $(\bar{v}, \bar{\xi}) \in \mathcal{Y}^{4+\alpha}\left(\overline{R_{0, T}}\right) \times$ $\mathcal{Z}^{1}[0, T]$ with $(\bar{v}(0, \cdot), \bar{\xi}(0))=\left(v_{0}(\cdot), \xi_{0}\right)$, we consider the linearized problem of $(3.2),(3.7)$ as follows:

$$
\left\{\begin{array}{l}
v_{t}=\mathcal{A}_{0} v+F_{0}(t, \eta) \quad \text { in } R_{0, T}, \\
v_{\eta}(t, 0)=\bar{\xi}(t) \tan \theta \\
v_{\eta}(t, 1)=0, \\
v_{\eta \eta \eta}(t, 0)=\frac{3 \tan \theta}{1+\tan ^{2} \theta} \cdot \frac{\bar{v}_{\eta \eta}^{2}(t, 0)}{\bar{\xi}(t)} \\
v_{\eta \eta \eta}(t, 1)=0 \\
v(0, \eta)=v_{0}(\eta)
\end{array}\right.
$$


Moreover, for $v$ satisfying (3.10), we consider the equation of the form

$$
\left\{\begin{array}{l}
\dot{\xi}(t)=-C_{1}(\theta) \frac{v_{\eta \eta \eta \eta}(t, 0)}{\bar{\xi}^{4}(t)}+C_{2}(\theta) \frac{v_{\eta \eta}^{3}(t, 0)}{\bar{\xi}^{6}(t)}, \\
\xi(0)=\xi_{0} .
\end{array}\right.
$$

REMARK 3.2 When we linearize (3.2)-(3.7), we should take care that the initial data $\Gamma_{0}$ is given in $C^{2+\alpha}$, and that the singularity of $\dot{\xi}$ at $t=0$ is expected to be equal to that of $v_{\eta \eta \eta \eta}$. So we first plug (3.8) into (3.2) and use $f$ as

$$
\begin{aligned}
f(\eta, & \left.v_{\eta}, v_{\eta \eta}, v_{\eta \eta \eta}, v_{\eta \eta \eta \eta}, \xi, v_{\eta \eta \eta \eta}(\cdot, 0), v_{\eta \eta}(\cdot, 0)\right) \\
= & -\frac{1}{\left(\xi^{2}+v_{\eta}^{2}\right)^{2}} v_{\eta \eta \eta \eta}+\frac{10 v_{\eta} v_{\eta \eta}}{\left(\xi^{2}+v_{\eta}^{2}\right)^{3}} v_{\eta \eta \eta}+\frac{3\left(\xi^{2}-5 v_{\eta}^{2}\right) v_{\eta \eta}^{3}}{\left(\xi^{2}+v_{\eta}^{2}\right)^{4}} \\
& +C_{1}(\theta) \frac{(1-\eta) v_{\eta}}{\xi^{5}} v_{\eta \eta \eta \eta}(\cdot, 0)-C_{2}(\theta) \frac{(1-\eta) v_{\eta}}{\xi^{7}} v_{\eta \eta}^{3}(\cdot, 0) .
\end{aligned}
$$

Then we linearize $f$ in (3.12) with respect to $v_{\eta \eta \eta}, v_{\eta \eta \eta \eta}, v_{\eta \eta \eta \eta}(\cdot, 0)$ around $\left(v_{0}, \xi_{0}\right)$ to get the linearized equation $v_{t}=\mathcal{A}_{0} v+F_{0}(t, \eta)$ in $R_{0, T}$. We should note that, owing to the third term of $\mathcal{A}_{0} v$, which is a contribution of the triple junction, the operator $\mathcal{A}_{0}$ is different from usual elliptic operators. For details, see the first half of the Appendix.

For the unique existence results of the problem (3.10) and the problem (3.11), we have the following lemma.

LEMMA 3.3 Let $\alpha \in(0,1)$ and let us assume that $(\bar{v}, \bar{\xi}) \in \mathcal{Y}^{4+\alpha}\left(\overline{R_{0, T}}\right) \times \mathcal{Z}^{1}[0, T]$ with $(\bar{v}(0, \cdot), \bar{\xi}(0))=\left(v_{0}(\cdot), \xi_{0}\right)$, which satisfies the assumption of Theorem 3.1. Then,

(i) there exists a unique solution $v \in \mathcal{Y}^{4+\alpha}\left(\overline{R_{0, T}}\right)$ of (3.10),

(ii) for $v$ obtained in (i), there exists a unique solution $\xi \in \mathcal{Z}^{1}[0, T]$ of (3.11).

This lemma is essentially obtained by the first half of the Appendix. Indeed, by virtue of that, we can apply the optimal regularity theory of analytic semigroups to the linearized problem (3.10), and (i) is proved (see [21]). We omit its detailed proof. Once (i) is verified, then (ii) is obvious.

Now we are ready to prove Theorem 3.1.

Proof of Theorem 3.1. We explain an outline of the proof. We carry out the detailed calculation in the Appendix.

In order to obtain local existence result for the full problem (3.2)-(3.9), we shall use a fixed point argument. So we let

$$
\begin{aligned}
\mathcal{D}:= & \left\{(v, \xi) \in \mathcal{Y}^{4+\alpha}\left(\overline{R_{0, T}}\right) \times \mathcal{Z}^{1}[0, T] ;\right. \\
& \left.(v(0, \cdot), \xi(0))=\left(v_{0}(\cdot), \xi_{0}\right),\|v\|_{\mathcal{Y}^{4+\alpha}\left(\overline{R_{0, T}}\right)}+\|\xi\|_{\mathcal{Z}^{1}[0, T]} \leqslant K\right\},
\end{aligned}
$$

for positive bounded parameters $K, T$ satisfying $K T^{1 / 2} \leqslant \xi_{0} / 4$. Here we note that, if $\xi \in \mathcal{D}$, then $\xi$ satisfies

$$
\xi_{0} / 2 \leqslant \xi(t) \leqslant 3 \xi_{0} / 2 \quad \text { for } t \in[0, T]
$$


by virtue of $K T^{1 / 2} \leqslant \xi_{0} / 4$. Moreover, we define the map $\Phi$ as

$$
\Phi: \mathcal{D} \ni(\bar{v}, \bar{\xi}) \mapsto(v, \xi) \in \mathcal{Y}^{4+\alpha}\left(\overline{R_{0, T}}\right) \times \mathcal{Z}^{1}[0, T],
$$

where $(v, \xi)$ is the unique solution of (3.10) and (3.11) established in Lemma 3.3. We shall show that, for a suitable constant $K$ and small enough $T$ depending on $\xi_{0}$ and $\left\|v_{0}\right\|_{C^{2+\alpha}(I)}$, the map $\Phi$ is contracting in $\mathcal{D}$. Once this is verified, then the map $\Phi$ has a unique fixed point in $\mathcal{D}$. Of course, this fixed point is a unique solution of the full problem (3.2)-(3.9) in $\mathcal{D}$.

We shall first prove that $\Phi$ maps $\mathcal{D}$ into itself when we choose suitable $K$ and $T$. By means of the Appendix, if $v$ satisfies the linearized problem (3.10), we get the estimate

$$
\|v\|_{\mathcal{Y}^{4+\alpha}\left(\overline{R_{0, T}}\right)} \leqslant M_{0}+N T^{v},
$$

where $M_{0}$ is a constant depending on $\xi_{0},\left\|v_{0}\right\|_{C^{2+\alpha}(I)}, \theta$, and $N$ is a constant depending on $\xi_{0},\left\|v_{0}\right\|_{C^{2+\alpha}(I)}, \theta, \alpha, \gamma(0<\alpha<\gamma<1), K$ (throughout this section any constant depending on the preceding quantities will be denoted by $N$ whose value may be different on each occasion), and $v=\min \{\alpha / 4,(\gamma-\alpha) / 4\} \in\left(0, \frac{1}{4}\right)$. In particular, we emphasize the dependences of $M_{0}$ on $\xi_{0},\left\|v_{0}\right\|_{C^{2+\alpha}(I)}$ and of $N$ on $K$. By virtue of the Appendix and the basic theory of elliptic equations, we can eventually check that $M_{0}$ is an increasing function of $1 / \xi_{0},\left\|v_{0}\right\|_{C^{2+\alpha}(I)}$, and that $N$ is an increasing function of $K$. Then, according to (3.11) and (3.14),

$$
\|\xi\|_{\mathcal{Z}^{1}[0, T]} \leqslant \xi_{0}+C_{1}(\theta)\left(\frac{2}{\xi_{0}}\right)^{4} M_{0}+N T^{v} .
$$

Hence, choosing

$$
K=2\left\{\xi_{0}+\left(1+16 C_{1}(\theta) \xi_{0}^{-4}\right) M_{0}\right\}
$$

and

$$
T_{0}=\min \left\{\left(\frac{K}{2 N}\right)^{1 / v},\left(\frac{\xi_{0}}{4 K}\right)^{2}\right\}
$$

we obtain that

$$
\|v\|_{\mathcal{Y}^{4+\alpha}\left(\overline{R_{0, T}}\right)}+\|\xi\|_{\mathcal{Z}^{1}[0, T]} \leqslant K \quad \text { for } T \leqslant T_{0}
$$

That is, $\Phi$ maps $\mathcal{D}$ into itself.

Next we prove that the map $\Phi$ is a contraction on $\mathcal{D}$ for a suitable choice of $T$. Let $\left(\bar{v}_{1}, \bar{\xi}_{1}\right),\left(\bar{v}_{2}, \bar{\xi}_{2}\right) \in \mathcal{D}$ with $T \leqslant T_{0}$, and put $\left(v_{1}, \xi_{1}\right)=\Phi\left(\bar{v}_{1}, \bar{\xi}_{1}\right),\left(v_{2}, \xi_{2}\right)=\Phi\left(\bar{v}_{2}, \bar{\xi}_{2}\right)$. Moreover, let $V=v_{1}-v_{2}, \Xi=\xi_{1}-\xi_{2}, \bar{V}=\bar{v}_{1}-\bar{v}_{2}, \bar{\Xi}=\bar{\xi}_{1}-\bar{\xi}_{2}$. Then the function $V$ satisfies

$$
\left\{\begin{array}{l}
V_{t}=\mathcal{A}_{0} V+\tilde{F}(t, \eta) \quad \text { in } R_{0, T}, \\
V_{\eta}(t, 0)=\bar{\Xi}(t) \tan \theta \\
V_{\eta}(t, 1)=0, \\
V_{\eta \eta \eta}(t, 0)=\frac{3 \tan \theta}{1+\tan ^{2} \theta}\left(b_{1}(t) \bar{V}_{\eta \eta}(t, 0)+b_{2}(t) \bar{\Xi}(t)\right) \\
V_{\eta \eta \eta}(t, 1)=0 \\
V(0, \eta)=0
\end{array}\right.
$$


and, for $V$ satisfying (3.18), the function $\Xi$ satisfies

$$
\left\{\begin{aligned}
& \frac{\mathrm{d}}{\mathrm{d} t} \Xi(t)=-\frac{C_{1}(\theta)}{\bar{\xi}_{1}^{4}(t)} V_{\eta \eta \eta \eta}(t, 0)+b_{3}(t) V_{\eta \eta}(t, 0) \\
&+\left[b_{4}(t) v_{2 \eta \eta \eta \eta}(t, 0)+b_{5}(t)\right] \bar{\Xi}(t), \\
& \Xi(0)=0,
\end{aligned}\right.
$$

where

$$
\begin{aligned}
\tilde{F}(t, \eta)= & \left(F_{0} \text { evaluated at } \bar{v}_{1} \text { and } \bar{\xi}_{1}\right)-\left(F_{0} \text { evaluated at } \bar{v}_{2} \text { and } \bar{\xi}_{2}\right) \\
= & -\left\{\frac{1}{\left(\bar{\xi}_{1}^{2}(t)+\bar{v}_{1 \eta}^{2}\right)^{2}}-\frac{1}{\left(\xi_{0}^{2}+v_{0 \eta}^{2}\right)^{2}}\right\} \bar{V}_{\eta \eta \eta \eta} \\
& +g_{1}\left(\bar{\xi}_{i}, \bar{v}_{i \eta}\right) \bar{v}_{2 \eta \eta \eta \eta} \bar{V}_{\eta}+g_{2}\left(\bar{\xi}_{i}, \bar{v}_{i \eta}\right) \bar{v}_{2 \eta \eta \eta \eta} \bar{\Xi}+\cdots,
\end{aligned}
$$

and $b_{1}(t), \ldots, b_{5}(t)$ are functions of $\bar{\xi}_{i}(t), \bar{v}_{i \eta \eta}(t, 0)(i=1,2)$ without significant singularities. Here, applying the Appendix again, we obtain

$$
\|V\|_{\mathcal{Y}^{4+\alpha}\left(\overline{R_{0, T}}\right)} \leqslant N T^{v}\left(\|\bar{V}\|_{\mathcal{Y}^{4+\alpha}\left(\overline{R_{0, T}}\right)}+\|\bar{\Xi}\|_{\mathcal{Z}^{1}[0, T]}\right) .
$$

Then, by means of (3.19) and (3.20),

$$
\|\Xi\|_{\mathcal{Z}^{1}[0, T]} \leqslant N T^{\mathcal{v}}\left(\|\bar{V}\|_{\mathcal{Y}^{4+\alpha}\left(\overline{R_{0, T}}\right)}+\|\bar{\Xi}\|_{\mathcal{Z}^{1}[0, T]}\right) .
$$

Thus we derive

$$
\|V\|_{\mathcal{Y}^{4+\alpha}\left(\overline{R_{0, T}}\right)}+\|\Xi\|_{\mathcal{Z}^{1}[0, T]} \leqslant N T^{v}\left(\|\bar{V}\|_{\mathcal{Y}^{4+\alpha}\left(\overline{R_{0, T}}\right)}+\|\bar{\Xi}\|_{\mathcal{Z}^{1}[0, T]}\right) .
$$

Consequently, $\Phi$ is a contraction on $\mathcal{D}$ for $T \leqslant T_{1}$, where

$$
T_{1}=\min \left\{\left(\frac{1}{2 N}\right)^{1 / v}, T_{0}\right\} .
$$

This completes the proof of Theorem 3.1.

REMARK 3.4 According to (3.15), (3.16) and (3.22), $T_{1}$ is an increasing function of its arguments. So, if we choose $\left(v\left(t_{0}, \cdot\right), \xi\left(t_{0}\right)\right)$ (where $t_{0}>0$ ) as the initial data, $T_{1}$ is an increasing function of $\xi\left(t_{0}\right), 1 /\left\|v\left(t_{0}, \cdot\right)\right\|_{C^{2+\alpha}(I)}$. Thus, as long as there exist constants $v_{1}, v_{2}$ such that

$$
0<v_{1} \leqslant \xi\left(t_{0}\right), \quad\left\|v\left(t_{0}, \cdot\right)\right\|_{C^{2+\alpha}(I)} \leqslant \nu_{2}<\infty,
$$

$T_{1}$ does not shrink to 0 . In fact,

$$
T_{1}\left(\xi\left(t_{0}\right), 1 /\left\|v\left(t_{0}, \cdot\right)\right\|_{C^{2+\alpha}(I)}\right) \geqslant T_{1}\left(v_{1}, 1 / v_{2}\right)>0 .
$$

This property is used in Section 4.

In the same way as we obtain the inequality (3.21), we can derive a uniqueness result. Since the proof can be done identically as in the derivation of (3.21), we only show its statement without the proof. 
PROPOSITION 3.5 (Uniqueness) Let $\tau_{0}(\geqslant 0)$ be a given constant, and let $\left(v_{\tau_{0}}, \xi_{\tau_{0}}\right)$ be given data satisfying $\left\|v_{\tau_{0}}\right\|_{C^{2+\alpha}(I)}<\infty$ and $\xi_{\tau_{0}}>0$ for $\alpha \in(0,1)$. Then, solutions of the problem (3.2)-(3.6) and (3.8) on the time interval $\left[\tau_{0}, \tau_{0}+T\right]$ with $T>0$ for the initial condition $\left(v\left(\tau_{0}, \cdot\right), \xi\left(\tau_{0}\right)\right)=$ $\left(v_{\tau_{0}}(\cdot), \xi_{\tau_{0}}\right)$ are unique in $\mathcal{Y}^{4+\alpha}\left(\overline{R_{\tau_{0}, \tau_{0}+T}}\right) \times \mathcal{Z}^{1}\left[\tau_{0}, \tau_{0}+T\right]$.

In the following we show that the solution obtained in Theorem 3.1 has a further regularity for $t \in\left(0, T_{1}\right]$.

THEOREM 3.6 (Regularity) Let $\alpha \in(0,1)$ and let $(v, \xi)$ be the solution obtained in Theorem 3.1. Then, $(v, \xi)$ also satisfies

$$
(v, \xi) \in C^{1,6+\alpha}\left(\left(0, T_{1}\right] \times I\right) \times C^{1+(2+\alpha) / 4}\left(0, T_{1}\right] .
$$

Proof. Let $\delta \in\left(0, T_{1}\right)$ be arbitrarily fixed. Then we study the problem (3.2)-(3.6) and (3.8) on the time interval $[\delta, \delta+T]$ with $T>0$ for the initial data $v(\delta, \cdot) \in C^{4+\alpha}(I)$ and $\xi(\delta)>0$, where $(v, \xi)$ is the solution obtained in Theorem 3.1. Now we set $R_{\delta+\varepsilon, \delta+T}=(\delta+\varepsilon, \delta+T] \times I$ for $\varepsilon \in(0, T)$, and define the spaces as

$$
\begin{gathered}
\mathcal{Y}^{6+\alpha}\left(\overline{R_{\delta, \delta+T}}\right):=\left\{w \in C^{1,4+\alpha}\left(\overline{R_{\delta, \delta+T}}\right) \cap C^{1,6+\alpha}\left(R_{\delta, \delta+T}\right) ;\|w\|_{\mathcal{Y}^{6+\alpha}\left(\overline{R_{\delta, \delta+T}}\right)}<\infty\right\}, \\
\mathcal{Z}^{(6+\alpha) / 4}[\delta, \delta+T]:=\left\{\zeta \in C^{1}[\delta, \delta+T] \cap C^{1+(2+\alpha) / 4}(\delta, \delta+T] ;\|\zeta\|_{\mathcal{Z}^{(6+\alpha) / 4}[\delta, \delta+T]}<\infty\right\},
\end{gathered}
$$

where the norms are defined by

$$
\begin{aligned}
\|w\|_{\mathcal{Y}^{6+\alpha}\left(\overline{R_{\delta, \delta+T}}\right)}:= & \|w\|_{C^{1,4+\alpha}\left(\overline{R_{\delta, \delta+T}}\right)}+\sup _{0<\varepsilon<T} \varepsilon^{1 / 4}\left\|\partial_{\eta}^{5} w\right\|_{C^{0, \alpha}\left(\overline{R_{\delta+\varepsilon, \delta+T}}\right)} \\
& +\sup _{0<\varepsilon<T} \varepsilon^{1 / 2}\left\|\partial_{\eta}^{6} w\right\|_{C^{0, \alpha}\left(\overline{R_{\delta+\varepsilon, \delta+T}}\right)} \\
& +\sup _{0<\varepsilon<T} \varepsilon^{1 / 2}\left\|w_{t}\right\|_{C^{0,2+\alpha}\left(\overline{R_{\delta+\varepsilon, \delta+T}}\right)} \\
\|\zeta\|_{\mathcal{Z}^{(6+\alpha) / 4}[\delta, \delta+T]}:= & \|\zeta\|_{C^{1}[\delta, \delta+T]}+\sup _{0<\varepsilon<T} \varepsilon^{1 / 2}[\dot{\zeta}]_{C^{(2+\alpha) / 4}[\delta+\varepsilon, \delta+T]} .
\end{aligned}
$$

We shall obtain the further regularity result via three steps.

Step 1 We shall first linearize the problem (3.2)-(3.6) and (3.8) about $(v(\delta, \cdot), \xi(\delta))$. For given $(\bar{w}, \bar{\zeta}) \in \mathcal{Y}^{6+\alpha}\left(\overline{R_{\delta, \delta+T}}\right) \times \mathcal{Z}^{(6+\alpha) / 4}[\delta, \delta+T]$ with $(\bar{w}(\delta, \cdot), \bar{\zeta}(\delta))=(v(\delta, \cdot), \xi(\delta))$, we consider the linearized problem as follows:

$$
\left\{\begin{array}{l}
w_{t}=\mathcal{A}_{\delta} w+F_{\delta}(t, \eta) \quad \text { in } R_{\delta, T}, \\
w_{\eta}(t, 0)=\bar{\zeta}(t) \tan \theta \\
w_{\eta}(t, 1)=0, \\
w_{\eta \eta \eta}(t, 0)=\frac{3 \tan \theta}{1+\tan ^{2} \theta} \cdot \frac{\bar{w}_{\eta \eta}^{2}(t, 0)}{\bar{\zeta}(t)}, \\
w_{\eta \eta \eta}(t, 1)=0 \\
w(\delta, \eta)=v(\delta, \eta) .
\end{array}\right.
$$

Moreover, for $w$ satisfying (3.23), we consider the problem

$$
\left\{\begin{array}{l}
\dot{\zeta}(t)=-C_{1}(\theta) \frac{w_{\eta \eta \eta \eta}(t, 0)}{\bar{\zeta}^{4}(t)}+C_{2}(\theta) \frac{w_{\eta \eta}^{3}(t, 0)}{\bar{\zeta}^{6}(t)} \\
\zeta(\delta)=\xi(\delta)
\end{array}\right.
$$


Then, using the same argument as we used to obtain Lemma 3.3, we can show that there exists a unique solution $(w, \zeta) \in \mathcal{Y}^{6+\alpha}\left(\overline{R_{\delta, \delta+T}}\right) \times \mathcal{Z}^{(6+\alpha) / 4}[\delta, \delta+T]$ of the linearized problem (3.23) and the problem (3.24).

Step 2 In order to use a fixed point argument, we let

$$
\begin{aligned}
\mathcal{E}:= & \left\{(w, \zeta) \in \mathcal{Y}^{6+\alpha}\left(\overline{R_{\delta, \delta+T}}\right) \times \mathcal{Z}^{(6+\alpha) / 4}[\delta, \delta+T] ;\right. \\
& \left.(w(\delta, \cdot), \zeta(\delta))=(v(\delta, \cdot), \xi(\delta)),\|w\|_{\mathcal{Y}^{6+\alpha}\left(\overline{R_{\delta, \delta+T}}\right)}+\|\zeta\|_{\mathcal{Z}^{(6+\alpha) / 4}[\delta, \delta+T]} \leqslant \tilde{K}\right\},
\end{aligned}
$$

for positive bounded parameters $\tilde{K}, T$ satisfying $\tilde{K} T \leqslant \xi(\delta) / 2$. Here we note that, if $\xi \in \mathcal{E}$, then $\xi$ satisfies $\xi(\delta) / 2 \leqslant \xi(t) \leqslant 3 \xi(\delta) / 2$ for any $t \in[\delta, \delta+T]$ by virtue of $\tilde{K} T \leqslant \xi(\delta) / 2$. Moreover, we define the map $\Phi_{\delta}$ as

$$
\Phi_{\delta}: \mathcal{E} \ni(\bar{w}, \bar{\zeta}) \mapsto(w, \zeta) \in \mathcal{Y}^{6+\alpha}\left(\overline{R_{\delta, \delta+T}}\right) \times \mathcal{Z}^{(6+\alpha) / 4}[\delta, \delta+T],
$$

where $w$ and $\zeta$ are the unique solutions of the problems (3.23) and (3.24) respectively. We claim that, for a suitable constant $\tilde{K}$ and small enough $T$, the map $\Phi_{\delta}$ is contracting on $\mathcal{E}$. Once this is verified, then a unique fixed point of $\Phi_{\delta}$ exists in $\mathcal{E}$. Of course, this fixed point is the desired solution of the problem (3.2)-(3.6) and (3.8) with the initial condition $(w(\delta, \cdot), \zeta(\delta))=(v(\delta, \cdot), \xi(\delta))$.

We verify this claim. Let us choose

$$
\begin{aligned}
\tilde{K} & =2\left(\xi(\delta)+M_{\delta}\right), \\
T_{2} & =\min \left\{\left(\frac{\tilde{K}}{2 \tilde{N}}\right)^{1 / v}, \frac{\xi(\delta)}{2 \tilde{K}},\left(\frac{1}{2 \tilde{N}}\right)^{1 / v}\right\}
\end{aligned}
$$

where $M_{\delta}$ is a constant depending on $\xi(\delta),\|v(\delta, \cdot)\|_{C^{4+\alpha}(I)}, \theta$, and $\tilde{N}$ is a constant depending on $\xi(\delta),\|v(\delta, \cdot)\|_{C^{4+\alpha}(I)}, \theta, \alpha, \gamma, \tilde{K}$. By the same argument about constants $M_{0}, N$ of the inequality (3.14), we can eventually check that $M_{\delta}$ is an increasing function of $1 / \xi(\delta),\|v(\delta, \cdot)\|_{C^{4+\alpha}(I)}$, and that $\tilde{N}$ is an increasing function of $\tilde{K}$. Then, in the same way as we obtain the inequality (3.17), we are led to

$$
\|w\|_{\mathcal{Y}^{6+\alpha}\left(\overline{R_{\delta, \delta+T}}\right)}+\|\zeta\|_{\mathcal{Z}^{(6+\alpha) / 4}[\delta, \delta+T]} \leqslant \tilde{K} \quad \text { for } T \leqslant T_{2}
$$

That is, $\Phi_{\delta}$ maps $\mathcal{E}$ into itself. The next task is to see that $\Phi_{\delta}$ is contracting in $\mathcal{E}$. To do so, let $\left(\bar{w}_{i}, \bar{\zeta}_{i}\right) \in \mathcal{E}(i=1,2)$ with $T \leqslant T_{2}$, and put $\left(w_{i}, \zeta_{i}\right)=\Phi_{\delta}\left(\bar{w}_{i}, \bar{\zeta}_{i}\right)(i=1,2)$. Then, in the same way as we obtain the inequality (3.21), we derive, for $T \leqslant T_{2}$,

$$
\begin{aligned}
& \left\|w_{1}-w_{2}\right\|_{\mathcal{Y}^{6+\alpha}\left(\overline{R_{\delta, \delta+T}}\right)}+\left\|\zeta_{1}-\zeta_{2}\right\|_{\mathcal{Z}^{(6+\alpha) / 4}[\delta, \delta+T]} \\
& \leqslant \frac{1}{2}\left(\left\|\bar{w}_{1}-\bar{w}_{2}\right\|_{\mathcal{Y}^{6+\alpha}\left(\overline{R_{\delta, \delta+T}}\right)}+\left\|\bar{\zeta}_{1}-\bar{\zeta}_{2}\right\|_{\mathcal{Z}^{(6+\alpha) / 4}[\delta, \delta+T]}\right),
\end{aligned}
$$

which shows that $\Phi_{\delta}$ is a contraction on $\mathcal{E}$. Thus the above claim is verified.

Consequently, for $T \in\left(0, T_{2}\right]$, there exists a unique solution $(w, \zeta) \in \mathcal{E}$ of the problem (3.2)(3.6) and (3.8) with the initial condition $(w(\delta, \cdot), \zeta(\delta))=(v(\delta, \cdot), \xi(\delta))$. Then, by virtue of Proposition 3.5, we see that this solution $(w, \zeta)$ coincides with $(v, \xi)$ obtained in Theorem 3.1. Thus, $(v, \xi)$ has the desired regularity in $\left[\delta, \delta+T_{2}\right]$ for any $\delta \in\left(0, T_{1}\right)$. 
Step 3 As far as $\delta+T_{2}<T_{1}$ for $\delta \in\left(0, T_{1}\right)$, we repeat the same procedure as in Step 2. Then we need an estimate concerning the infimum of $T_{2}$. In order to see this, we use (3.13) and (3.17) to get, for $T_{1}$ obtained in Theorem 3.1,

$$
\xi(t) \geqslant \frac{\xi_{0}}{2}>0, \quad\|v(t, \cdot)\|_{C^{4+\alpha}(I)} \leqslant K \delta^{-1 / 2} \quad \text { for } t \in\left[\delta, T_{1}\right]
$$

where $K$ is the constant established by (3.15). So, by means of (3.26), we obtain

$$
T_{2}\left(\xi(t), 1 /\|v(t, \cdot)\|_{C^{4+\alpha}(I)}\right) \geqslant T_{2}\left(\xi_{0} / 2, \delta^{1 / 2} / K\right)>0 \quad \text { for } t \in\left[\delta, T_{1}\right]
$$

that is, as long as $t \in\left[\delta, T_{1}\right], T_{2}$ evaluated at $\xi(t)$ and $1 /\|v(t, \cdot)\|_{C^{4+\alpha}(I)}$ does not shrink to 0 . Consequently, $(v, \xi)$ has the desired regularity in $\left(0, T_{1}\right]$ since $\delta$ is arbitrary in $\left(0, T_{1}\right)$. This completes the proof of Theorem 3.6.

REMARK 3.7 In the same way as we obtained Theorem 3.6, we see that the solution obtained in Theorem 3.1 also belongs to $C^{1,2 m+2+\alpha}\left(\left(0, T_{1}\right] \times I\right) \times C^{(2 m+2+\alpha) / 4}\left(0, T_{1}\right]$ for any positive integer $m$ and $\alpha \in(0,1)$.

\section{Global existence}

The purpose of this section is to obtain global solutions of (3.2)-(3.9) and consequently of (1.3), (1.4) when the initial data $\Gamma_{0}$ and $\Gamma_{\theta}, A_{0}$ are sufficiently close to each other in some sense. The procedure to prove this is as follows. First we show that if the above condition is fulfilled, then the derivative of the curvature $\kappa$ of $\Gamma^{3}(t), \kappa_{s}$, is always small (in this and the remaining sections, for simplicity, we omit the upper indices 3 of $\kappa^{3}$ and $V^{3}$ ). In this step we need $C^{6}$-regularity for solutions of (3.2)-(3.9). Of course, this requirement is fulfilled by virtue of Theorem 3.6. Next, using this result, we derive a priori estimates of the solutions $(v(t, \eta), \xi(t))$ of (3.2)-(3.9) in a suitable norm and we then obtain the desired global existence result.

In order to derive an a priori estimate we employ a similar method to those of X. Chen [5] and C. M. Elliott and H. Garcke [8], although they only studied the motion of closed curves. In our case the question is how to deal with the motion of the triple junction. We shall settle this issue to establish a modified version of the estimates obtained in [5] and [8].

First we mention the energy-decreasing and the area-preserving properties found by Garcke and Novick-Cohen [15] in the general setting in (1.1), (1.2). In this case the energy $\tilde{E}$ associated with (1.1), (1.2) is defined by

$$
\tilde{E}[\Gamma(t)]:=\sum_{i=1}^{3} \sigma^{i} L\left[\Gamma^{i}(t)\right]
$$

(see [15]), where $L\left[\Gamma^{i}(t)\right]$ denotes the length of $\Gamma^{i}(t)$. Let $i, j, k \in\{1,2,3\}$ be mutually different. Let $\Omega^{i}(t)$ be the domain enclosed by $\Gamma^{j}(t), \Gamma^{k}(t)$, and $\partial \Omega$, where we assume that $\Gamma^{1}(t), \Gamma^{2}(t)$, and $\Gamma^{3}(t)$ are found in counterclockwise order. Let $\left|\Omega^{i}(t)\right|$ be the area of $\Omega^{i}(t)$. 
Lemma 4.1 ( [15]) Assume that $\Gamma(\cdot)$ be the $C^{1,4}$-solution of (1.1), (1.2) with $\Gamma_{0} \in C^{3}$ satisfying suitable compatibility conditions. Then, it holds for $t \geqslant 0$

$$
\begin{aligned}
& \frac{\mathrm{d} \tilde{E}[\Gamma(t)]}{\mathrm{d} t}=-\sum_{i=1}^{3}\left(\sigma^{i}\right)^{2} l^{i} \int_{0}^{L\left[\Gamma^{i}(t)\right]}\left(\kappa_{s}^{i}\right)^{2} \mathrm{~d} s, \\
& \left|\Omega^{i}(t)\right|=\left|\Omega^{i}(0)\right| .
\end{aligned}
$$

Proof. The explicit proof of (4.1) is given in [15]. Here we provide a proof of (4.2). We carry out this only for $i=1$, since the cases for $i=2,3$ can be done identically.

Let $V_{\partial \Omega^{1}(t)}$ be the outward normal velocity of $\partial \Omega^{1}(t)$. Then we have

$$
\frac{\mathrm{d}\left|\Omega^{1}(t)\right|}{\mathrm{d} t}=\int_{\partial \Omega^{1}(t)} V_{\partial \Omega^{1}(t)} \mathrm{d} s
$$

(see [18: Section 2]), where $s$ runs clockwise. Recall that $\partial \Omega^{1}(t)=\Gamma^{3}(t) \cup \partial \Omega \cup \Gamma^{2}(t)$. Taking into account both the direction of $s$ on $\Gamma^{2}(t)$ and the fact that $V_{\partial \Omega^{1}(t)} \equiv 0$ when $s \in \partial \Omega$, we obtain

$$
\frac{\mathrm{d}\left|\Omega^{1}(t)\right|}{\mathrm{d} t}=\int_{0}^{L\left[\Gamma^{3}(t)\right]} V^{3} \mathrm{~d} s-\int_{0}^{L\left[\Gamma^{2}(t)\right]} V^{2} \mathrm{~d} s .
$$

Plugging (A) in (1.1) into the above yields

$$
\frac{\mathrm{d}\left|\Omega^{1}(t)\right|}{\mathrm{d} t}=l^{3} \sigma^{3} \kappa_{s}^{3}(t, 0)-l^{2} \sigma^{2} \kappa_{s}^{2}(t, 0)-l^{3} \sigma^{3} \kappa_{s}^{3}\left(t, L\left[\Gamma^{3}(t)\right]\right)+l^{2} \sigma^{2} \kappa_{s}^{2}\left(t, L\left[\Gamma^{2}(t)\right]\right) .
$$

By the balance of fluxes at $s=0$ and the no flux condition at $s=L\left[\Gamma^{i}(t)\right]$ for $i=2,3$, the last terms vanish. This completes the proof.

In the following we set for simplicity

$$
L(t)=L\left[\Gamma^{3}(t)\right]
$$

Let $\Gamma(t)$ be the solution of (1.3) with the initial data $\Gamma_{0} \in \mathcal{S}_{\theta}$, which has enough regularity such that at least $\Gamma(\cdot) \in C^{1,4}$ for $t>0$. Let $A(t)$ be the area enclosed by $\Gamma^{3}(t)$, the $x$-axis, and the $y$-axis, and also let $A_{0}$ be the area enclosed by $\Gamma_{0}^{3}$, the $x$-axis, and the $y$-axis. Then, in our symmetric setting Lemma 4.1 reads

$$
\begin{gathered}
\frac{\mathrm{d} E[\Gamma(t)]}{\mathrm{d} t}=-\int_{0}^{L(t)} \kappa_{s}(t, s)^{2} \mathrm{~d} s \\
A(t)=A_{0}
\end{gathered}
$$

Next we show a priori estimates for $E[\Gamma(t)]$ and $L\left[\Gamma^{3}(t)\right]$.

Proposition 4.2 For all $t \geqslant 0$ it holds that

$$
\begin{aligned}
& E\left[\Gamma_{\theta, A}\right] \leqslant E[\Gamma(t)] \leqslant E\left[\Gamma_{0}\right], \\
& \sqrt{\pi A_{0}} \leqslant L(t) \leqslant E\left[\Gamma_{0}\right] .
\end{aligned}
$$


Proof. We show (4.6). The first inequality is due to Theorem 2.1 and the second inequality is due to (4.4), which shows (4.6).

Next we show (4.7). The first inequality is due to Lemma 2.3 and the second inequality follows from the inequality $L(t) \leqslant E[\Gamma(t)]$ and (4.6).

In the following let $\Gamma(t)$ be the solution of (1.3), (1.4) with the regularity in Theorem 3.6. We investigate the boundary values of the derivatives of the curvature $\kappa$.

LEMMA 4.3 There holds for $t>0$

(i) $\kappa_{s s}(t, 0)=-\frac{\mathrm{d} \xi}{\mathrm{d} t} \sin \theta$

(ii) $\kappa_{s s s}(t, 0)=-\kappa(t, 0) \frac{\mathrm{d} \xi}{\mathrm{d} t} \cos \theta$,

(iii) $\kappa_{s s s}(t, L(t))=0$.

Proof. (i) Differentiating the equality $u(t,-\xi(t))=0$ with respect to $t$ and using the condition $u_{x}(t,-\xi(t))=\tan \theta$, we get

$$
u_{t}(t,-\xi(t))=\frac{\mathrm{d} \xi}{\mathrm{d} t} \tan \theta
$$

On the other hand, from the equation $V=-\kappa_{s s}$ we have

$$
\kappa_{s s}(t, s)=-\frac{u_{t}}{\left(1+u_{x}^{2}\right)^{1 / 2}}(t, x) .
$$

Here the relation between $s$ and $x$ is

$$
s=\int_{-\xi(t)}^{x}\left(1+u_{x}^{2}\right)^{1 / 2}\left(t, x^{\prime}\right) \mathrm{d} x^{\prime} .
$$

Now, evaluating (4.9) at $s=0$ and using the equality $u_{x}(t,-\xi(t))=\tan \theta$ and (4.8), we obtain (i).

(ii) Differentiating (4.9) with respect to $s$ and using $\partial / \partial s=\left(1+u_{x}^{2}\right)^{-1 / 2} \partial / \partial x$, we have

$$
\kappa_{s s s}(t, s)=\left(-\frac{u_{t x}}{1+u_{x}^{2}}+\frac{u_{t} u_{x} u_{x x}}{\left(1+u_{x}^{2}\right)^{2}}\right)(t, x) .
$$

On the other hand, differentiating the equality $u_{x}(t,-\xi(t))=\tan \theta$ with respect to $t$, we get

$$
u_{t x}(t,-\xi(t))=u_{x x}(t,-\xi(t)) \frac{\mathrm{d} \xi}{\mathrm{d} t}
$$

Evaluating (4.11) at $s=0$ and using (4.12) and the condition $u_{x}(t,-\xi(t))=\tan \theta$, we get (ii).

(iii) It follows from the condition $u_{x}(t, 0)=0$ that $u_{t x}(t, 0)=0$. With this in mind we evaluate (4.11) at $s=L(t)$ to get (iii). 
We are going to obtain an a priori estimate for $\kappa_{s}$ in $L^{2}\left(\Gamma^{3}(t)\right)$ by taking care of the boundary values obtained in Lemma 4.3 and employing the method as in [5] and [8]. For this purpose we use a new parametrization of $\Gamma^{3}(t)$ as

$$
\Gamma^{3}(t)=\left\{\hat{F}(t, p) \in \mathbb{R}^{2} ; p \in\left[p_{0}(t), p_{1}(t)\right]\right\}
$$

satisfying

$$
\begin{aligned}
& \varphi:\left[p_{0}(t), p_{1}(t)\right] \ni p \mapsto x \in[-\xi(t), 0]: \text { monotone increasing, } \\
& \hat{F}\left(t, p_{0}(t)\right)=(-\xi(t), 0), \quad \hat{F}\left(t, p_{1}(t)\right)=(0, u(t, 0)), \\
& \hat{F}_{t} \cdot \hat{F}_{p}=0 \quad \text { for } t \geqslant 0 \text { and } p \in\left[p_{0}(t), p_{1}(t)\right],
\end{aligned}
$$

where - denotes the standard inner product in $\mathbb{R}^{2}$. We write the curvature $\kappa(t, s)$ and the normal velocity $V(t, s)$ in this new coordinate as

$$
\hat{\kappa}(t, p)=\kappa(t, s), \quad \hat{V}(t, p)=V(t, s) .
$$

Here $p$ and $s$ are linked via $\varphi$ and (4.10). Then $\hat{\kappa}(t, p)$ satisfies the identity

$$
\hat{\kappa}_{t}=\Delta \hat{V}+(\hat{\kappa})^{2} \hat{V},
$$

where

$$
\Delta \hat{V}:=\frac{1}{\hat{g}}\left(\hat{V}_{p p}-\frac{1}{2} \frac{\hat{g}_{p}}{\hat{g}} \hat{V}_{p}\right), \quad \hat{g}(t, p):=\hat{F}_{p}(t, p) \cdot \hat{F}_{p}(t, p)
$$

(see [18: Section 2]). Since the equation $V=-\kappa_{S S}$ is equivalent to the one $\hat{V}=-\Delta \hat{\kappa}$, we get the equation

$$
\hat{\kappa}_{t}=-\Delta^{2} \hat{\kappa}-\hat{\kappa}^{2} \Delta \hat{\kappa} \quad \text { for } t \geqslant 0 \text { and } p \in\left[p_{0}(t), p_{1}(t)\right] .
$$

We multiply (4.13) by $-2 \Delta \hat{\kappa}$ and integrate it on $\Gamma^{3}(t)$. Then a straightforward computation gives

$$
\begin{aligned}
& -\int_{p_{0}(t)}^{p_{1}(t)} 2 \Delta \hat{\kappa} \cdot \hat{\kappa}_{t} \sqrt{\hat{g}} \mathrm{~d} p=\frac{\mathrm{d}}{\mathrm{d} t} \int_{0}^{L(t)} \kappa_{s}^{2} \mathrm{~d} s+\int_{0}^{L(t)} \kappa \kappa_{s}^{2} \kappa_{s s} \mathrm{~d} s, \\
& \int_{p_{0}(t)}^{p_{1}(t)} 2 \Delta \hat{\kappa}\left(\Delta^{2} \hat{\kappa}+\hat{\kappa}^{2} \Delta \hat{\kappa}\right) \sqrt{\hat{g}} \mathrm{~d} p=\int_{0}^{L(t)} 2 \kappa_{s s}\left(\kappa_{s s s s}+\kappa^{2} \kappa_{s s}\right) \mathrm{d} s .
\end{aligned}
$$

Thus we have

$$
\begin{aligned}
\frac{\mathrm{d}}{\mathrm{d} t}\left\|\kappa_{s}(t)\right\|_{2}^{2}= & -\int_{0}^{L(t)} \kappa \kappa_{s}^{2} \kappa_{s s} \mathrm{~d} s+2 \int_{0}^{L(t)} \kappa_{s s} \kappa_{s s s s} \mathrm{~d} s \\
& +2 \int_{0}^{L(t)} \kappa^{2} \kappa_{s s}^{2} \mathrm{~d} s \\
= & : J_{1}+J_{2}+J_{3} .
\end{aligned}
$$

Here and hereafter we set for simplicity

$$
\|\cdot\|_{2}=\|\cdot\|_{L^{2}\left(\Gamma^{3}(t)\right)}, \quad\|\cdot\|_{\infty}=\|\cdot\|_{L^{\infty}\left(\Gamma^{3}(t)\right)} .
$$

We estimate $J_{i}, i=1,2,3$. 
LEMMA 4.4 There holds for $t>0$

$$
\begin{aligned}
\left|J_{1}\right| \leqslant & \left(L(t)^{3}\left\|\kappa_{s}(t)\right\|_{2}^{2}+\theta L(t)^{3 / 2}\left\|\kappa_{s}(t)\right\|_{2}\right)\left\|\kappa_{s s s}(t)\right\|_{2}^{2}, \\
J_{2} \leqslant & 2\left(\frac{L(t)^{3 / 2}}{\tan \theta}\left\|\kappa_{s}(t)\right\|_{2}+\frac{\theta}{\tan \theta}-1\right)\left\|\kappa_{s s s}(t)\right\|_{2}^{2}, \\
J_{3} \leqslant & 2\left(L(t)^{3}\left\|\kappa_{s}(t)\right\|_{2}^{2}+2 \theta L(t)^{3 / 2}\left\|\kappa_{s}(t)\right\|_{2}+\delta_{1}\right)\left\|\kappa_{s s s}(t)\right\|_{2}^{2} \\
& +C\left(\delta_{1}\right) \theta^{4} L(t)^{-4}\left\|\kappa_{s}(t)\right\|_{2}^{2},
\end{aligned}
$$

where $\delta_{1}>0$ is arbitrary small constant and $C\left(\delta_{1}\right)$ is a constant depending on $\delta_{1}$.

Proof. We use the following inequalities, which are easily derived by the fact that $\kappa_{s}=0$ at $s=0$ and $L(t)$ :

$$
\begin{aligned}
& \left\|\left(\kappa-\kappa_{a v}\right)(t)\right\|_{\infty} \leqslant L(t)^{1 / 2}\left\|\kappa_{s}(t)\right\|_{2}, \\
& \left\|\partial_{S}^{j} \kappa(t)\right\|_{2} \leqslant L(t)\left\|\partial_{s}^{j+1} \kappa(t)\right\|_{2}, \\
& \left\|\partial_{S}^{j} \kappa(t)\right\|_{\infty} \leqslant L(t)^{1 / 2}\left\|\partial_{S}^{j+1} \kappa(t)\right\|_{2}
\end{aligned}
$$

for $j=1,2$; here $\kappa_{a v}(t)$ is the averaged curvature of $\kappa(t, s)$ defined by

$$
\kappa_{a v}(t)=\frac{1}{L(t)} \int_{0}^{L(t)} \kappa(t, s) \mathrm{d} s .
$$

We also note that

$$
\kappa_{a v}(t)=-\frac{\theta}{L(t)}
$$

Indeed, if we let $\omega(t, s)$ be the angle of the unit normal of $\Gamma^{3}(t)$ from the $x$-axis at $s$, then $\kappa(t, s)=$ $\partial \omega / \partial s(t, s)$. It then follows from (4.21) that

$$
\kappa_{a v}(t)=\frac{1}{L(t)} \int_{0}^{L(t)} \frac{\partial \omega}{\partial s} \mathrm{~d} s=\frac{1}{L(t)}(\omega(t, L(t))-\omega(t, 0)) .
$$

Note that the boundary conditions $u_{x}(t,-\xi(t))=\tan \theta$ and $u_{x}(t, 0)=0$ mean that $\omega(t, 0)=$ $\pi / 2+\theta$ and $\omega(t, L(t))=\pi / 2$, respectively. Thus we obtain (4.22).

To show (4.15), we use (4.22) to get

$$
\begin{aligned}
\left|J_{1}\right| & \leqslant \int_{0}^{L(t)}\left|\kappa-\kappa_{a v}\right|\left|\kappa_{s}\right|^{2}\left|\kappa_{s s}\right| \mathrm{d} s+\int_{0}^{L(t)}\left|\kappa_{a v} \| \kappa_{s}\right|^{2}\left|\kappa_{s s}\right| \mathrm{d} s \\
& \leqslant\left(\left\|\kappa-\kappa_{a v}\right\|_{\infty}\left\|\kappa_{s}\right\|_{2}^{2}\left\|\kappa_{s s}\right\|_{\infty}+\frac{\theta}{L}\left\|\kappa_{s}\right\|_{2}^{2}\left\|\kappa_{s s}\right\|_{\infty}\right)(t) .
\end{aligned}
$$

Now (4.18)-(4.20) yield (4.15).

We show (4.16). An integration by parts and Lemma 4.3 imply

$$
J_{2}=-2 \sin \theta \cos \theta \kappa(t, 0)\left(\frac{\mathrm{d} \xi}{\mathrm{d} t}\right)^{2}-2\left\|\kappa_{s s s}(t)\right\|_{2}^{2} .
$$


It also follows from Lemma 4.3(i) and (4.20) with $j=2$ that

$$
\left(\frac{\mathrm{d} \xi}{\mathrm{d} t}\right)^{2}=\frac{1}{\sin ^{2} \theta} \kappa_{s s}(t, 0)^{2} \leqslant \frac{L(t)}{\sin ^{2} \theta}\left\|\kappa_{s s s}(t)\right\|_{2}^{2} .
$$

Then the above inequality together with (4.18) and (4.22) yields

$$
\begin{aligned}
& \left|\sin \theta \cos \theta \kappa(t, 0)\left(\frac{\mathrm{d} \xi}{\mathrm{d} t}\right)^{2}\right| \\
& \leqslant \frac{L(t)}{\tan \theta}\left(\left|\kappa(t, 0)-\kappa_{a v}(t)\right|+\left|\kappa_{a v}(t)\right|\right)\left\|\kappa_{s s s}(t)\right\|_{2}^{2} \\
& \leqslant \frac{1}{\tan \theta}\left(L(t)^{3 / 2}\left\|\kappa_{s}(t)\right\|_{2}+\theta\right)\left\|\kappa_{s s s}(t)\right\|_{2}^{2} .
\end{aligned}
$$

We plug this into (4.23) to get (4.16).

We show (4.17). We split $J_{3}$ as

$$
\begin{aligned}
J_{3}= & 2 \int_{0}^{L(t)}\left|\kappa-\kappa_{a v}\right|^{2} \kappa_{s s}^{2} \mathrm{~d} s+4 \kappa_{a v} \int_{0}^{L(t)}\left(\kappa-\kappa_{a v}\right) \kappa_{s s}^{2} \mathrm{~d} s \\
& +2 \kappa_{a v}^{2} \int_{0}^{L(t)} \kappa_{s s}^{2} \mathrm{~d} s \\
= & : J_{3,1}+J_{3,2}+J_{3,3} .
\end{aligned}
$$

Applying (4.18), (4.19) with $j=2$, and (4.22) to $J_{3,1}$ and $J_{3,2}$, we have

$$
\begin{aligned}
& J_{3,1} \leqslant 2 L(t)^{3}\left\|\kappa_{s}(t)\right\|_{2}^{2}\left\|\kappa_{s s s}(t)\right\|_{2}^{2}, \\
& J_{3,2} \leqslant 4 \theta L(t)^{3 / 2}\left\|\kappa_{s}(t)\right\|_{2}\left\|\kappa_{s s s}(t)\right\|_{2}^{2} .
\end{aligned}
$$

An integration by parts in $J_{3,3}$ employing the condition that $\kappa_{s}=0$ at $s=0$ and $L(t)$ yields

$$
\begin{aligned}
J_{3,3} & =-2 \kappa_{a v}(t)^{2} \int_{0}^{L(t)} \kappa_{s} \kappa_{s s s} \mathrm{~d} s \leqslant 2 \kappa_{a v}(t)^{2}\left\|\kappa_{s}(t)\right\|_{2}\left\|\kappa_{s s s}(t)\right\|_{2} \\
& \leqslant \frac{1}{2 \delta_{1}}\left(\frac{\theta}{L(t)}\right)^{4}\left\|\kappa_{s}(t)\right\|_{2}^{2}+2 \delta_{1}\left\|\kappa_{s s s}(t)\right\|_{2}^{2} .
\end{aligned}
$$

Thus (4.17) is proved.

It is observed that $1-\theta / \tan \theta>0$ when $\theta \in(0, \pi / 2)$. Hence we can choose $\delta_{1}>0$ so small so that $1-\theta / \tan \theta-\delta_{1}>0$. We fix such a $\delta_{1}$ and put

$$
H(l, \lambda)=2\left(1-\frac{\theta}{\tan \theta}-\delta_{1}\right)-\left\{3 l^{3} \lambda^{2}+\left(5 \theta+\frac{2}{\tan \theta}\right) l^{3 / 2} \lambda\right\} \quad \text { for } l, \lambda>0 .
$$

Then from Lemma 4.4 and (4.14) we get

$$
\begin{aligned}
& \frac{\mathrm{d}}{\mathrm{d} t}\left\|\kappa_{s}(t)\right\|_{2}^{2}+H\left(L(t),\left\|\kappa_{s}(t)\right\|_{2}\right)\left\|\kappa_{s s S}(t)\right\|_{2}^{2} \\
& \leqslant C\left(\delta_{1}\right) \theta^{4} L(t)^{-4}\left\|\kappa_{s}(t)\right\|_{2}^{2} .
\end{aligned}
$$


Integrating this on $[0, t]$ and using Proposition 4.2 and (4.4), we arrive at

$$
\begin{aligned}
& \left\|\kappa_{s}(t)\right\|_{2}^{2}+\int_{0}^{t} H\left(E\left[\Gamma_{0}\right],\left\|\kappa_{s}(\tau)\right\|_{2}\right)\left\|\kappa_{s s s}(\tau)\right\|_{2}^{2} \mathrm{~d} \tau \\
& \leqslant\left\|\kappa_{0, s}\right\|_{2}^{2}+C_{\theta, A_{0}}\left(E\left[\Gamma_{0}\right]-E\left[\Gamma_{\theta, A_{0}}\right]\right) .
\end{aligned}
$$

Here we put

$$
C_{\theta, A_{0}}=C\left(\delta_{1}\right) \theta^{4}\left(\sqrt{\pi A_{0}}\right)^{-4} .
$$

Now we are ready to show an a priori estimate of $\kappa_{s}$ when $\Gamma_{0}$ and $\Gamma_{\theta, A_{0}}$ are sufficiently close to each other. To make the statement precise, we put

$$
\rho_{0}^{2}:=\left\|\kappa_{0, s}\right\|_{2}^{2}+C_{\theta, A_{0}}\left(E\left[\Gamma_{0}\right]-E\left[\Gamma_{\theta, A_{0}}\right]\right) .
$$

Then we say that $\Gamma_{0}$ and $\Gamma_{\theta, A_{0}}$ are close to each other if $H\left(E\left[\Gamma_{0}\right], \rho_{0}\right)>0$. The following proposition establishes a modified version of the a priori estimate presented in [5] and [8].

Proposition 4.5 The following two statements hold.

(i) If $E\left[\Gamma_{0}\right]=E\left[\Gamma_{\theta}, A_{0}\right]$, then $\Gamma(t)=\Gamma_{\theta}, A_{0}$ for $t \geqslant 0$.

(ii) If $E\left[\Gamma_{0}\right]>E\left[\Gamma_{\theta}, A_{0}\right]$ and $H\left(E\left[\Gamma_{0}\right], \rho_{0}\right)>0$, then

$$
\left\|\kappa_{s}(t)\right\|_{2}^{2}+H\left(E\left[\Gamma_{0}\right], \rho_{0}\right) \int_{0}^{t}\left\|\kappa_{s s s}(\tau)\right\|_{2}^{2} \mathrm{~d} \tau \leqslant \rho_{0}^{2} \quad \text { for } t \geqslant 0 .
$$

REMARK 4.6 Proposition 4.5 together with (4.18) and (4.7) shows that if $\Gamma_{0}$ and $\Gamma_{\theta, A_{0}}$ are close to each other, then $\kappa(t, s)$ is close to $\kappa_{a v}(t)$ for $t \geqslant 0$ and $s \in[0, L(t)]$.

Proof of Proposition 4.5. (i) If $E\left[\Gamma_{0}\right]=E\left[\Gamma_{\theta, A_{0}}\right]$, then Theorem 2.1 implies that $\Gamma_{0}=\Gamma_{\theta, A_{0}}$. Since $\Gamma_{\theta, A_{0}}$ is a stationary solution of (1.3) and Proposition 3.5, on the other hand, guarantees the uniqueness of the solution of (1.3), (1.4), we conclude that the solution $\Gamma(t)$ of (1.3) with $\Gamma_{0}=\Gamma_{\theta, A_{0}}$ must coincide with $\Gamma_{\theta, A_{0}}$ for all $t \geqslant 0$.

(ii) If $E\left[\Gamma_{0}\right]>E\left[\Gamma_{\theta, A_{0}}\right]$, the proof can proceed as in [8] by virtue of the boundary condition $\kappa_{s}=0$ at $s=0$ and $L(t)$, so we omit the details.

By virtue of Proposition 4.5 we can obtain a number of a priori estimates. The contributions arising from the motion of the triple junction emerge as various functions of $\theta$. Throughout the remaining part of this paper we always assume the following conditions on initial data $\Gamma_{0}$ :

$$
\begin{aligned}
E\left[\Gamma_{0}\right]>E\left[\Gamma_{\theta, A_{0}}\right], & H\left(E\left[\Gamma_{0}\right], \rho_{0}\right)>0, \\
\theta+E\left[\Gamma_{0}\right]^{3 / 2} \rho_{0}<\pi / 2, & \sqrt{\pi A_{0}} \sin \theta / \theta-\frac{1}{2} E\left[\Gamma_{0}\right]^{5 / 2} \rho_{0}>0 .
\end{aligned}
$$

The reason why the third and fourth conditions are imposed will be clarified in Remarks 4.8 and 4.12 .

We begin by showing an a priori estimate of $\omega(t, s)$, the angle of the unit normal of $\Gamma^{3}(t)$ from the $x$-axis at $s$. 
PROPOSITION 4.7 It holds that

$$
\frac{\pi}{2}-E\left[\Gamma_{0}\right]^{3 / 2} \rho_{0} \leqslant \omega(t, s) \leqslant \frac{\pi}{2}+\theta+E\left[\Gamma_{0}\right]^{3 / 2} \rho_{0}
$$

for $t \geqslant 0$ and $s \in[0, L(t)]$.

REMARK 4.8 Owing to the third assumption in (4.28), the right-hand side of (4.29) is less than $\pi$, which means that $\Gamma^{3}(t)$ does not develop graph-breaking.

Proof of Proposition 4.7. We only show the estimtate from below. The estimate from above can be checked almost identically.

Since $\partial \omega / \partial s=\kappa$, we have

$$
\begin{aligned}
\omega(t, s) & =\frac{\pi}{2}+\theta+\int_{0}^{s} \kappa(t, \sigma) \mathrm{d} \sigma \\
& =\frac{\pi}{2}+\theta+\kappa_{a v}(t) s+\int_{0}^{s}\left(\kappa(t, \sigma)-\kappa_{a v}(t)\right) \mathrm{d} \sigma .
\end{aligned}
$$

We use (4.22) in the third term and (4.18) in the last term. Then,

$$
\omega(t, s) \geqslant \frac{\pi}{2}+\theta\left(1-\frac{s}{L(t)}\right)-L(t)^{3 / 2}\left\|\kappa_{s}(t)\right\|_{2} .
$$

Now (4.7) and (4.27) yield the desired result.

LEMMA 4.9 It holds that

$$
-\left(E\left[\Gamma_{0}\right]^{1 / 2} \rho_{0}+\frac{\theta}{\sqrt{\pi A_{0}}}\right) \leqslant \kappa(t, s) \leqslant E\left[\Gamma_{0}\right]^{1 / 2} \rho_{0}-\frac{\theta}{E\left[\Gamma_{0}\right]}
$$

for $t \geqslant 0$ and $s \in[0, L(t)]$.

Proof. We write

$$
\kappa(t, s)=\left(\kappa(t, s)-\kappa_{a v}(t)\right)+\kappa_{a v}(t) .
$$

Now a similar argument employed in the proof of Proposition 4.7 gives (4.30).

Now we shall derive an a priori estimate for $\xi(t)$. Let $(X, Y)(t, s)$ be the parametrization of $\Gamma^{3}(t)$ with respect to its arc-length parameter $s$. Then the unit tangent of $\Gamma^{3}(t)$ at $s$ is given by $(\sin \omega(t, s),-\cos \omega(t, s))$. Thus we have

$$
(X, Y)(t, s)=\left(-\xi(t)+\int_{0}^{s} \sin \omega(t, \sigma) \mathrm{d} \sigma,-\int_{0}^{s} \cos \omega(t, \sigma) \mathrm{d} \sigma\right) .
$$

Let $\Gamma_{a v}^{3}(t)$ be the circular arc in $\{(x, y) ; x \leqslant 0, y \geqslant 0\}$ with the radius $\left|\kappa_{a v}(t)\right|^{-1}=L(t) / \theta$ and with the centre on the $y$-axis and with $\left.\angle\left(\Gamma_{a v}^{3}(t), x\right.$-axis $)\right|_{x=-\xi_{a v}(t)}=\theta$, where $-\xi_{a v}(t)$ is the $x$ coordinate of the point at which $\Gamma_{a v}^{3}(t)$ intersects to the $x$-axis. Then it is easily seen that the total length of $\Gamma_{a v}^{3}(t)$ is also equal to $L(t)$. Let $\left(X_{a v}, Y_{a v}\right)(t, s) \in \mathbb{R}^{2}$ be the parametrization of $\Gamma_{a v}^{3}(t)$ with its arc-length parameter $s \in[0, L(t)]$. Let $\omega_{a v}(t, s)$ be the angle of the unit normal of $\Gamma_{a v}^{3}(t)$ from the $x$-axis at $s$. Then, as in (4.31), we have

$$
\left(X_{a v}, Y_{a v}\right)(t, s)=\left(-\xi_{a v}(t)+\int_{0}^{s} \sin \omega_{a v}(t, \sigma) \mathrm{d} \sigma,-\int_{0}^{s} \cos \omega_{a v}(t, \sigma) \mathrm{d} \sigma\right) .
$$

The following lemma enables us to relate $\Gamma^{3}(t)$ with $\Gamma_{a v}^{3}(t)$. 
LEMMA 4.10 There holds for $t \geqslant 0$ and $s \in[0, L(t)]$

$$
\begin{gathered}
\left|X(t, s)-X_{a v}(t, s)\right| \leqslant \frac{1}{2} L(t)^{5 / 2}\left\|\kappa_{s}(t)\right\|_{2}, \\
\left|Y(t, s)-Y_{a v}(t, s)\right| \leqslant \frac{1}{2} L(t)^{5 / 2}\left\|\kappa_{s}(t)\right\|_{2} .
\end{gathered}
$$

Proof. We employ the method as in [5]. Note the facts that

$$
\omega_{a v}(t, 0)=\frac{\pi}{2}+\theta, \quad \xi_{a v}(t)=L(t) \frac{\sin \theta}{\theta},
$$

which are easily seen by an elementary geometric observation. Since

$$
X(t, L(t))=X_{a v}(t, L(t))=0,
$$

we have

$$
\xi(t)=\int_{0}^{L(t)} \sin \omega(t, \sigma) \mathrm{d} \sigma, \quad \xi_{a v}(t)=\int_{0}^{L(t)} \sin \omega_{a v}(t, \sigma) \mathrm{d} \sigma .
$$

On the other hand, we integrate the identities $\partial \omega / \partial s=\kappa$ and $\partial \omega_{a v} / \partial s=\kappa_{a v}$ with respect to $s$. By virtue of the first of (4.35) and (4.18), we have

$$
\begin{aligned}
\left|\omega(t, s)-\omega_{a v}(t, s)\right| & \leqslant \int_{0}^{s}\left|\kappa(t, \sigma)-\kappa_{a v}(t)\right| \mathrm{d} \sigma \\
& \leqslant L(t)^{1 / 2}\left\|\kappa_{s}\right\|_{2} s .
\end{aligned}
$$

Then, by (4.36) and (4.37),

$$
\begin{aligned}
\left|X(t, s)-X_{a v}(t, s)\right| & =\left|\int_{L(t)}^{s} \sin \omega(t, \sigma) \mathrm{d} \sigma-\int_{L(t)}^{s} \sin \omega_{a v}(t, \sigma) \mathrm{d} \sigma\right| \\
& \leqslant \int_{s}^{L(t)}\left|\omega(t, \sigma)-\omega_{a v}(t, \sigma)\right| \mathrm{d} \sigma \\
& \leqslant L(t)^{1 / 2}\left\|\kappa_{S}(t)\right\|_{2} \int_{s}^{L(t)} \sigma \mathrm{d} \sigma \\
& \leqslant \frac{1}{2} L(t)^{5 / 2}\left\|\kappa_{s}(t)\right\|_{2} .
\end{aligned}
$$

Thus we have proved (4.33). A similar argument as in (4.33) gives (4.34).

The following proposition establishes a priori bounds for the triple junction.

PROPOSITION 4.11 It holds that

$$
\sqrt{\pi A_{0}} \frac{\sin \theta}{\theta}-\frac{1}{2} E\left[\Gamma_{0}\right]^{5 / 2} \rho_{0} \leqslant \xi(t) \leqslant E\left[\Gamma_{0}\right] \frac{\sin \theta}{\theta}+\frac{1}{2} E\left[\Gamma_{0}\right]^{5 / 2} \rho_{0}
$$

for $t \geqslant 0$. 
REMARK 4.12 Owing to the fourth assumption in (4.28), it is assured that $\xi(t)>0$ for $t \geqslant 0$.

Proof of Proposition 4.11. It follows from (4.35) and (4.7) that

$$
\sqrt{\pi A_{0}} \frac{\sin \theta}{\theta} \leqslant \xi_{a v}(t) \leqslant E\left[\Gamma_{0}\right] \frac{\sin \theta}{\theta} .
$$

Evaluating (4.33) at $s=0$ and using (4.7), (4.27), and the above inequality, we get (4.38).

Now we can show an a priori estimate of $v$.

Proposition 4.13 Let $\alpha \in(0,1 / 2]$. Then we have

$$
\|v(t)\|_{C^{2+\alpha}(I)} \leqslant B_{0} \quad \text { for } t \geqslant 0 .
$$

Here $B_{0}>0$ is a constant depending only on $\Gamma_{0}$ through $\rho_{0}, A_{0}$, and $E\left[\Gamma_{0}\right]$.

Proof. First we differentiate $v(t, \eta)=Y(t, s)$ with respect to $\eta$ to get

$$
v_{\eta}=Y_{s} \cdot\left(\xi^{2}+v_{\eta}^{2}\right)^{1 / 2} \text {. }
$$

On the other hand, by (4.31), $Y_{s}=-\cos \omega$. Thus

$$
v_{\eta}^{2}=\xi^{2} \frac{\cos ^{2} \omega}{\sin ^{2} \omega} .
$$

It then follows from (4.29) and (4.38) that there is a constant $B_{0,1}$ depending on $\Gamma_{0}$ such that

$$
\left\|v_{\eta}(t)\right\|_{C(I)} \leqslant B_{0,1} \quad \text { for } t \geqslant 0 .
$$

Moreover, since the condition $v(t, 0)=0$ yields the estimate $|v(t, \eta)| \leqslant\left\|v_{\eta}(t)\right\|_{C(I)}$, we obtain

$$
\|v(t)\|_{C^{1}(I)} \leqslant B_{0,1} \quad \text { for } t \geqslant 0 .
$$

Next we differentiate (4.40) with respect to $\eta$. With the equality $Y_{s s}=\kappa \sin \omega$ in mind we obtain

$$
v_{\eta \eta}=\frac{\kappa \sin \omega \cdot\left(\xi^{2}+v_{\eta}^{2}\right)}{1+v_{\eta} \cos \omega \cdot\left(\xi^{2}+v_{\eta}^{2}\right)^{-1 / 2}} .
$$

Now (4.30), (4.38), and (4.41) imply that there is a constant $B_{0,2}$ depending on $\Gamma_{0}$ such that

$$
\left\|v_{\eta \eta}(t)\right\|_{C(I)} \leqslant B_{0,2} \quad \text { for } t \geqslant 0 .
$$

Finally, we show that there is a constant $B_{0,3}$ depending on $\Gamma_{0}$ such that

$$
\left[v_{\eta \eta}(t, \cdot)\right]_{C^{\alpha}[0,1]} \leqslant B_{0,3} \quad \text { for } t \geqslant 0 \text { with } \alpha \in\left(0, \frac{1}{2}\right] .
$$

Indeed, differentiating (4.40) twice with respect to $\eta$ and using $Y_{s s s}=\kappa_{s} \sin \omega+\kappa^{2} \cos \omega$, we have

$$
\begin{aligned}
& \left(1+\frac{v_{\eta} \cos \omega}{\left(\xi^{2}+v_{\eta}^{2}\right)^{1 / 2}}\right) v_{\eta \eta \eta} \\
& =\left(\kappa_{s} \sin \omega+\kappa^{2} \cos \omega\right)\left(\xi^{2}+v_{\eta}^{2}\right)^{3 / 2}+3 \kappa v_{\eta} v_{\eta \eta} \sin \omega \\
& \quad-\frac{\xi^{2} v_{\eta \eta}^{2} \cos \omega}{\left(\xi^{2}+v_{\eta}^{2}\right)^{3 / 2}} .
\end{aligned}
$$


We take the $L^{2}(I)$-norm in both sides and use (4.30), (4.38), (4.41), and (4.42). Consequently, we can find a constant $B_{0,3}$ depending on $\Gamma_{0}$ such that

$$
\left\|v_{\eta \eta \eta}(t)\right\|_{L^{2}(I)} \leqslant B_{0,3} \quad \text { for } t \geqslant 0 .
$$

Now (4.43) immediately follows from the above inequality when $\alpha \in\left(0, \frac{1}{2}\right]$.

Now by virtue of Propositions 4.11 and 4.13 one can always solve (3.2)-(3.9) on the time intervals $\left[0, T_{*}\right],\left[T_{*}, 2 T_{*}\right],\left[2 T_{*}, 3 T_{*}\right], \ldots$, for a $T_{*}>0$ which is determined only on $\Gamma_{0}$. Thus, we arrive at the following global existence result.

THEOREM 4.14 (Global existence) Let $\alpha \in(0,1 / 2]$. Assume (4.28). Then the equations (3.2)(3.9) admits a unique global solution $(v, \xi) \in \mathcal{Y}^{4+\alpha}\left(\overline{R_{0, T}}\right) \times \mathcal{Z}^{1}[0, T]$ for any $T>0$. Consequently, the problem (1.1), (1.2) admits a unique global solution in $C^{1+\alpha / 4,4+\alpha}$ for $t>0$ provided the initial data belong to $\mathcal{S}_{\theta}$ and are also close to $\Gamma_{\theta, A_{0}}$.

REMARK 4.15 The global solutions obtained in Theorem 4.14 also possess the additional regularities guaranteed in Theorem 3.6 and Remark 3.7 with $\alpha \in\left(0, \frac{1}{2}\right]$.

\section{Convergence to the minimizer of the energy}

This section gives the proofs that the global solution of (1.3), (1.4) obtained in Theorem 4.14 converges to the minimizer $\Gamma_{\theta, A_{0}}$ of the energy $E$ and the energy of the solution converges to $E\left[\Gamma_{\theta, A_{0}}\right]$ as $t \rightarrow \infty$.

The fundamental tool to obtain this result is the following fact.

Lemma 5.1 Assume that $\Gamma(t)=\bigcup_{i=1}^{3} \Gamma^{i}(t)$ is a (at least) $C^{6}$-global solution of (1.3), (1.4) with a $C^{3}$-initial data $\Gamma_{0} \in \mathcal{S}_{\theta}$. Let $\kappa(t, s)$ be the curvature of $\Gamma^{3}(t)$. Then,

$$
\left\|\kappa_{s}(t)\right\|_{2} \rightarrow 0 \quad \text { as } t \rightarrow \infty .
$$

This lemma can be proved as in [8: Theorem 6.4] by employing the same argument to derive (4.24), so we omit its proof.

Let $\Gamma(t)$ be the global solution of (1.3), (1.4) for the initial data $\Gamma_{0} \in \mathcal{S}_{\theta}$ constructed in Theorem 4.14. Put

$$
\Gamma_{a v}(t)=\bigcup_{i=1}^{3} \Gamma_{a v}^{i}(t)
$$

where

$$
\begin{aligned}
& \Gamma_{a v}^{1}(t)=\left\{(x, 0) ; x \in\left[-a,-\xi_{a v}(t)\right]\right\}, \\
& \Gamma_{a v}^{2}(t)=\left\{\left(X_{a v},-Y_{a v}\right)(t, s) ; s \in[0, L(t)]\right\}, \\
& \Gamma_{a v}^{3}(t)=\left\{\left(X_{a v}, Y_{a v}\right)(t, s) ; s \in[0, L(t)]\right\}
\end{aligned}
$$

for $t \geqslant 0$ and $\left(X_{a v}, Y_{a v}\right)(t, s)$ is given in (4.32). 
Lemma 5.2 Put $E_{\star}:=\lim _{t \rightarrow \infty} E[\Gamma(t)]$ and $L_{\star}:=\theta\left(E_{\star}-a \cos \theta\right) /(\theta-\sin \theta \cos \theta)$. Then,

$$
\begin{aligned}
& E\left[\Gamma_{a v}(t)\right] \rightarrow E_{\star}, \\
& L(t) \rightarrow L_{\star}
\end{aligned}
$$

as $t \rightarrow \infty$.

Proof. Since $E\left[\Gamma_{a v}(t)\right]=\left(a-\xi_{a v}(t)\right) \cos \theta+L(t)$, we have

$$
E\left[\Gamma_{a v}(t)\right]-E[\Gamma(t)]=\left(\xi(t)-\xi_{a v}(t)\right) \cos \theta .
$$

Then (4.33) at $s=0$ implies

$$
\left|\xi(t)-\xi_{a v}(t)\right| \leqslant \frac{1}{2} L(t)^{5 / 2}\left\|\kappa_{S}(t)\right\|_{2} \leqslant \frac{1}{2} E\left[\Gamma_{0}\right]^{5 / 2}\left\|\kappa_{s}(t)\right\|_{2}
$$

Moreover, from Lemma 5.1 it follows that the right-hand side converges to 0 as $t \rightarrow \infty$. Thus we get (5.2).

We check (5.3). By (4.35) we have

$$
E\left[\Gamma_{a v}(t)\right]=a \cos \theta+\left(1-\frac{\sin \theta \cos \theta}{\theta}\right) L(t) .
$$

Using (5.2) and passing to the limit as $t \rightarrow \infty$ in the above equality, we get (5.3).

The next lemma shows that the large-time profile of $\Gamma(t)$ is approximated by $\Gamma_{a v}(t)$.

LEMMA 5.3

$$
\lim _{t \rightarrow \infty} \sup _{s \in[0, L(t)]}\left(\left|X-X_{a v}\right|+\left|Y-Y_{a v}\right|\right)(t, s)=0 .
$$

Proof. It follows from (4.33) and (4.34) that

$$
\sup _{s \in[0, L(t)]}\left(\left|X-X_{a v}\right|+\left|Y-Y_{a v}\right|\right)(t, s) \leqslant L(t)^{5 / 2}\left\|\kappa_{s}(t)\right\|_{2} .
$$

Now (5.3) and Lemma 5.1 give the desired result.

We put

$$
\xi_{\star}:=L_{\star} \frac{\sin \theta}{\theta}, \quad \kappa_{\star}:=-\frac{\theta}{L_{\star}} .
$$

Define a circular arc in $\{(x, y) ; x \leqslant 0, y \geqslant 0\}$ by

$$
\left(X_{\star}, Y_{\star}\right)(s)=\left(-\xi_{\star}+\int_{0}^{s} \sin \left(\frac{\pi}{2}+\theta+\kappa_{\star} \sigma\right) \mathrm{d} \sigma,-\int_{0}^{s} \cos \left(\frac{\pi}{2}+\theta+\kappa_{\star} \sigma\right) \mathrm{d} \sigma\right)
$$

for $s \in\left[0, L_{\star}\right]$ and set

$$
\Gamma_{\star}=\bigcup_{i=1}^{3} \Gamma_{\star}^{i} \in \mathcal{S}_{\theta},
$$


where

$$
\begin{aligned}
\Gamma_{\star}^{1} & =\left\{(x, 0) ; x \in\left[-a,-\xi_{\star}\right]\right\}, \\
\Gamma_{\star}^{2} & =\left\{\left(X_{\star},-Y_{\star}\right)(s) ; s \in\left[0, L_{\star}\right]\right\}, \\
\Gamma_{\star}^{3} & =\left\{\left(X_{\star}, Y_{\star}\right)(s) ; s \in\left[0, L_{\star}\right]\right\} .
\end{aligned}
$$

The next lemma shows that the large-time profile of $\Gamma_{a v}(t)$ is described by $\Gamma_{\star}$.

LEMMA 5.4

$$
\Gamma_{a v}(t) \rightarrow \Gamma_{\star} \quad \text { as } t \rightarrow \infty
$$

Proof. Passing to the limit as $t \rightarrow \infty$ in the second equation in (4.35) and (4.22), we have

$$
\lim _{t \rightarrow \infty} \xi_{a v}(t)=\xi_{\star}, \quad \lim _{t \rightarrow \infty} \kappa_{a v}(t)=\kappa_{\star} .
$$

Then, by the first equation in (4.35),

$$
\begin{aligned}
\omega_{a v}(t, s) & =\omega_{a v}(t, 0)+\int_{0}^{s} \frac{\partial \omega_{a v}}{\partial \sigma}(t, \sigma) \mathrm{d} \sigma \\
& =\frac{\pi}{2}+\theta+\kappa_{a v}(t) s \rightarrow \frac{\pi}{2}+\theta+\kappa_{\star} s \quad \text { as } t \rightarrow \infty
\end{aligned}
$$

for $s \in\left[0, L_{\star}\right]$. Now, passing to the limit as $t \rightarrow \infty$ in (4.32), we obtain the desired result.

Thus we arrive at the desired result on the convergence of the solution as $t \rightarrow \infty$.

\section{THEOREM 5.5}

$$
\Gamma(t) \rightarrow \Gamma_{\theta, A_{0}} \quad \text { as } t \rightarrow \infty .
$$

Proof. By virtue of Lemmas 5.3 and 5.4 we get

$$
\Gamma(t) \rightarrow \Gamma_{\star} \quad \text { as } t \rightarrow \infty
$$

Let $A_{\star}$ be the area enclosed by $\Gamma_{\star}^{3}$, the $x$-axis, and the $y$-axis. If $\Gamma_{\star} \neq \Gamma_{\theta, A_{0}}$, then $A_{\star}$ is not equal to $A_{0}$. On the other hand (4.5) and (5.6) imply that $A_{\star}$ must coincide with $A_{0}$. This yields a contradiction and we then conclude that $\Gamma_{\star}$ must coincide with $\Gamma_{\theta}, A_{0}$. This completes the proof.

We can also show the convergences of both the length and the energy of the solution as $t \rightarrow \infty$. The key point is to make use of the area-preserving property.

TheOREM 5.6 We have

$$
\begin{aligned}
& \lim _{t \rightarrow \infty} L\left[\Gamma^{3}(t)\right]=L\left[\Gamma_{\theta, A_{0}}^{3}\right], \\
& \lim _{t \rightarrow \infty} E[\Gamma(t)]=E\left[\Gamma_{\theta, A_{0}}\right] .
\end{aligned}
$$

Proof. We first show (5.7). We use the following fact. 
Fact. Let $D \subset \mathbb{R}^{2}$ be a simply connected bounded domain with the piecewise smooth boundary $\partial D$ with the total length $L$. Suppose that $\partial D$ is parameterized by its arc-length parameter $s$ running clockwise as $\partial D=\left\{F(s) \in \mathbb{R}^{2} ; s \in[0, L]\right\}$. Let $v(s)$ be the outward unit normal field on $\partial D$. Then,

$$
\text { the area of } D=\frac{1}{2} \int_{0}^{L} F(s) \cdot v(s) \mathrm{d} s .
$$

We apply the formula (5.9) to the domain enclosed by $\Gamma^{3}(t)$, the $y$-axis, and the $x$-axis to get

$$
\begin{aligned}
A(t)= & \frac{1}{2} \int_{0}^{L(t)}(X, Y)(t, s) \cdot(\cos \omega, \sin \omega)(t, s) \mathrm{d} s \\
& +\frac{1}{2} \int_{u(t, 0)}^{0}(0, y) \cdot(1,0) \mathrm{d} y+\frac{1}{2} \int_{0}^{-\xi(t)}(x, 0) \cdot(0,-1) \mathrm{d} x .
\end{aligned}
$$

The last two terms of the right-hand side vanish. We use (4.5) in the left-hand side and use (4.31) with (4.36) in the first term of the right-hand side. Moreover, after using Fubini's theorem, we have

$$
A_{0}=-\int_{0}^{L(t)}\left(\int_{0}^{s} \cos \omega(t, \sigma) \mathrm{d} \sigma\right) \sin \omega(t, s) \mathrm{d} s .
$$

On the other hand, using (4.37), (4.7), and Lemma 5.1, we get

$$
\left|\omega(t, s)-\omega_{a v}(t, s)\right| \leqslant E\left[\Gamma_{\theta, A_{0}}\right]^{3 / 2}\left\|\kappa_{s}(t)\right\|_{2} \rightarrow 0 \quad \text { as } t \rightarrow \infty
$$

for $s \in\left[0, L_{\star}\right]$. Recalling (5.5) and (5.4), we have

$$
\omega_{a v}(t, s) \rightarrow \frac{\pi}{2}+\theta-\frac{\theta}{L_{\star}} s \quad \text { as } t \rightarrow \infty
$$

for $s \in\left[0, L_{\star}\right]$. Consequently, we have

$$
\omega(t, s) \rightarrow \frac{\pi}{2}+\theta-\frac{\theta}{L_{\star}} s \quad \text { as } t \rightarrow \infty
$$

for $s \in\left[0, L_{\star}\right]$. Now we let $t \rightarrow \infty$ in (5.10) and calculate the integral to obtain

$$
L_{\star}=r_{\theta, A_{0}} \theta,
$$

where $r_{\theta, A_{0}}$ is defined in (1.5). This shows (5.7).

Now (5.8) is a direct consequence of (5.11) and the definition of $L_{\star}$ in the statement of Lemma 5.2. This completes the proof.

\section{Acknowledgement}

The authors are grateful to Professor Y. Giga for suggesting the present problem and continuing encouragement. They also thank the referees for their valuable comments which made it possible to improve the previous version of this paper. The first author is partially supported by The Japan Society for Promotion of Science through Grant No. 10740079. 


\section{REFERENCES}

1. Acquistapace, P. \& Terreni, B. Hölder classes with boundary conditions as interpolation spaces. Math. Z. 195, (1987) 451-471.

2. Bronsard, L. \& Reitich, F. On three-phase boundary motion and the singular limit of a vector-valued Ginzburg-Landau equation. Arch. Rat. Mech. Anal. 124, (1993) 355-379.

3. Cahn, J. W., Elliott, C. M., \& Novick-Cohen, A. The Cahn-Hilliard equation with a concentration dependent mobility: motion by minus the Laplacian of the mean curvature. European $J$. Appl. Math. 7, (1996) 287-301.

4. CAHn, J. W. \& TAYlor, J. E. Surface motion by surface diffusion. Acta Metall. 42, (1994) 1045-1063.

5. CHEN, X. The Hele-Shaw problem and area-preserving curve-shortening motions. Arc. Rat. Mech. Anal. 123, (1993) 117-151.

6. Сhор, D. L. \& Sethinan, J. A. Motion by intrinsic Laplacian of curvature. Interfaces and Free Boundaries 1, (1999) 107-123.

7. Davi, F. \& Gurtin, M. E. On the motion of a phase interface by surface diffusion. Z. Angew. Math. Phys. 41, (1990) 782-811.

8. Elliott, C. M. \& Garcke, H. Existence results for diffusive surface motion laws. Advances in Mathematical Sciences and Applications 7, (1997) 467-490.

9. Elliott, C. M. \& Maier-PAape, S. Losing a graph with surface diffusion. Hokkaido Math. J. to appear.

10. Escher, J., MAYer, U. F., \& Simonett, G. The surface diffusion flow for immersed hypersurfraces. SIAM J. Math. Anal. 29, (1998) 1419-1433.

11. Escher, J., Mayer, U. F., \& Simonett, G. On the Surface Diffusion Flow, Navier-Stokes Equations and Related Nonlinear Problems (Palanga, 1997). VSP, Utrecht (1998) pp. 69-79.

12. Escher, J. \& SimonetT, G. Moving surfaces and abstract parabolic evolution equations. In: Escher, J. \& Simonett, G. (eds), Topics in Nonlinear Analysis, The Herbert Amann Anniversary Volume. pp. 183-212. PNLDE 35, Birkhäuser, (1999).

13. Escher, J. \& Simonett, G. The volume preserving mean curvature flow near spheres. Proc. Am. Math. Soc. 126, (1998) 2789-2796.

14. Galaktionov, V. A., Hulshof, J., \& VazQuez, J. L. Extinction and focusing behaviour of spherical and annular flames described by a free boundary problem. J. Math. Pure Appl. 76, (1997) 563-608.

15. GARCKe, H. \& Novick-Cohen, A. A singular limit for a system of degenerate Cahn-Hilliard equations. Adv. Diff. Eqns. 5, (2000) 401-434.

16. Giga, Y. \& ItO, K. On pinching of curves moved by surface diffusion. Comm. Appl. Anal. 2, (1998) 393-405.

17. GIGA, Y. \& ITO, K. Loss of convexity of simple closed curves moved by surface diffusion. In: EsCHER, J. \& Simonett, G. (eds), Topics in Nonlinear Analysis, The Herbert Amann Anniversary Volume. pp. 305-320. PNLDE 35, Birkhäuser, (1999).

18. Gurtin, M. E. Thermomechanics of Evolving Phase Boundaries in the Plane. Clarendon Press, Oxford (1993).

19. Hilhorst, D. \& Hulshof, J. A free boundary focusing problem. Proc. Am. Math. Soc. 121, (1994) 1193-1202.

20. Iто, K. Loss of convexity of compact hypersurfaces moved by surface diffusion. Comm. Appl. Anal. 6, (2002).

21. Lunardi, A. Analytic Semigroups and Optimal Regularity in Parabolic Problems. Birkhäuser, (1995).

22. Lunardi, A., Sinestrari, E., \& Von Wahl, W. A semigroup approach to the time dependent parabolic initial-boundary value problem. Diff. Int. Eqns. 5, (1992) 1275-1306.

23. Mayer, U. F. \& Simonett, G. Self-intersections for the surface diffusion and the volume-preserving mean curvature flow. Diff. Int. Eqns. 13, (2000) 1189-1199.

24. Mullins, W. W. Theory of thermal grooving. J. Appl. Phys. 28, (1957) 333-339. 
25. Solonnikov, V. A. Boundary value problems in physics. Proceedings of the Steklov Institute of Mathematics. 83, (1965).

26. STEWART, H. B. Generation of analytic semigroups by strongly elliptic operators under general boundary conditions. Trans. Am. Math. Soc. 259, (1980) 299-310.

27. Sternberg, P. \& Ziemer, W. P. Local minimisers of a three-phase partition problem with triple junctions. Proc. R. Soc. Edinburgh 124 A, (1994) 1059-1073.

\section{Appendix}

In this section we shall prove the inequalities (3.14) and (3.20). To prove them, we use the optimal regularity theory of analytic semigroups as comprehensively studied in [21].

Here we summarize a basic abstract framework used below. Let $X$ be a Banach space with norm $\|\cdot\|$ and let $A: D(A) \subset X \rightarrow X$ be a linear operator satisfying the following condition:

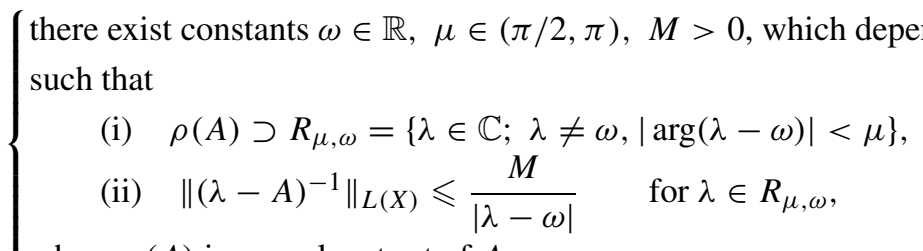

where $\rho(A)$ is a resolvent set of $A$.

If $A$ satisfies the condition (A.1), $A$ is said to be sectorial in $X$. Then $A$ generates an analytic semigroup $\mathrm{e}^{t A}$ in $X$ for $t \geqslant 0$. In addition, a family of intermediate spaces between $D(A)$ and $X$ can be defined by

$$
D_{A}(\beta, \infty)=\left\{\phi \in X ;[\phi]_{D_{A}(\beta, \infty)}=\sup _{0<t \leqslant 1}\left\|t^{1-\beta} A \mathrm{e}^{t A} \phi\right\|<\infty\right\}, \quad 0<\beta<1 .
$$

They are Banach spaces under the norm

$$
\|\phi\|_{D_{A}(\beta, \infty)}=\|\phi\|+[\phi]_{D_{A}(\beta, \infty)} .
$$

Moreover, the following estimate is known. For $k \in \mathbb{N}, \beta_{1}, \beta_{2} \in(0,1)$, there exists a constant $C=C\left(k, \beta_{1}, \beta_{2}, A\right)$ such that

$$
\left\|t^{k-\beta_{1}+\beta_{2}} A^{k} \mathrm{e}^{t A}\right\|_{L\left(D_{A}\left(\beta_{1}, \infty\right), D_{A}\left(\beta_{2}, \infty\right)\right)} \leqslant C \quad \text { for } 0<t \leqslant 1 .
$$

The statement holds also for $k=0$, provided that $\beta_{1} \leqslant \beta_{2}$.

We shall return to our problem. In the remaining part of this section, we use $C_{\alpha}, C_{\theta}$, etc. to denote various constants. Here their subindices are written to emphasize what they depend on. Let $\tau \geqslant 0$. We define two differential operators $\mathcal{A}_{\tau}^{(1)}$ and $\mathcal{A}_{\tau}^{(2)}$, for $C^{4}$-function $U:[0,1] \ni \eta \mapsto$ $U(\eta) \in \mathbb{R}$ and given $(\bar{v}, \bar{\xi}) \in \mathcal{Y}^{4+\alpha}\left(\overline{R_{\tau, \tau+T}}\right) \times \mathcal{Z}^{1}[\tau, \tau+T]$, by

$$
\begin{aligned}
& \mathcal{A}_{\tau}^{(1)} U:=-\frac{1}{\left(\bar{\xi}^{2}(\tau)+\bar{v}_{\eta}^{2}(\tau, \eta)\right)^{2}} \partial_{\eta}^{4} U+10 \frac{\bar{v}_{\eta}(\tau, \eta) \bar{v}_{\eta \eta}(\tau, \eta)}{\left(\bar{\xi}^{2}(\tau)+\bar{v}_{\eta}^{2}(\tau, \eta)\right)^{3}} \partial_{\eta}^{3} U, \\
& \mathcal{A}_{\tau}^{(2)} U:=C_{1}(\theta)(1-\eta) \frac{\bar{v}_{\eta}(\tau, \eta)}{\bar{\xi}^{5}(\tau)} \partial_{\eta}^{4} U(0) .
\end{aligned}
$$


If we define $X:=C[0,1]$ and

$$
D\left(A_{\tau}^{(1)}\right):=\left\{U \in C^{4}[0,1] ; \partial_{\eta} U(0)=\partial_{\eta} U(1)=\partial_{\eta}^{3} U(0)=\partial_{\eta}^{3} U(1)=0\right\},
$$

then $A_{\tau}^{(1)}: \quad X \supset D\left(A_{\tau}^{(1)}\right) \ni U \mapsto \mathcal{A}_{\tau}^{(1)} U \in X$ is the realization of $\mathcal{A}_{\tau}^{(1)}$ in $X$ and satisfies the condition (A.1) where constants $\omega, \mu, M$ depend on $\bar{\xi}(\tau),\|\bar{v}(\tau, \cdot)\|_{C^{2+\alpha}[0,1]}$ (see [26]). Therefore, $A_{\tau}^{(1)}$ is sectorial in $X$. In particular, we note that these constants increase with $1 / \bar{\xi}(\tau),\|\bar{v}(\tau, \cdot)\|_{C^{2+\alpha}[0,1]}$. Moreover, we obtain the following equalities:

$$
D_{A_{\tau}^{(1)}}(\beta, \infty)= \begin{cases}C^{4 \beta}[0,1], & \text { if } 0<\beta<\frac{1}{4}, \\ C_{b_{1}}^{4 \beta}[0,1], & \text { if } \frac{1}{4}<\beta<\frac{3}{4}, \\ C_{b_{2}}^{4 \beta}[0,1], & \text { if } \frac{3}{4}<\beta<1 .\end{cases}
$$

with equivalence of norms (see, for example, $[1,22]$ ), where

$$
\begin{aligned}
& C_{b_{1}}^{4 \beta}[0,1]:=\left\{U \in C^{4 \beta}[0,1] ; \partial_{\eta} U(0)=\partial_{\eta} U(1)=0\right\}, \\
& C_{b_{2}}^{4 \beta}[0,1]:=\left\{U \in C^{4 \beta}[0,1] ; \partial_{\eta} U(0)=\partial_{\eta} U(1)=\partial_{\eta}^{3} U(0)=\partial_{\eta}^{3} U(1)=0\right\} .
\end{aligned}
$$

It can be checked that the constants, which assure equivalence of norms, increase with $1 / \bar{\xi}(\tau),\|\bar{v}(\tau, \cdot)\|_{C^{2+\alpha}[0,1]}$. From now on we represent the constants concerned with equivalence of norms as $C_{e q}$. Here $\beta=\frac{1}{4}$ and $\beta=\frac{3}{4}$ are sensitive cases. But these cases are unnecessary in our paper, so we don't make mention of them.

Now let $A_{\tau}^{(2)}: X \supset D\left(A_{\tau}^{(1)}\right) \ni U \mapsto \mathcal{A}_{\tau}^{(2)} U \in X$ be the realization of $\mathcal{A}_{\tau}^{(2)}$ in $X$. Then we are led to the following lemma.

Lemma A. 1 Let $A_{\tau}:=A_{\tau}^{(1)}+A_{\tau}^{(2)}$. Then,

(i) $A_{\tau}$ is a sectorial operator in $X$.

(ii) there exists a constant $C$, which increases with $1 / \bar{\xi}(\tau),\|\bar{v}(\tau, \cdot)\|_{C^{2+\alpha}[0,1]}$, such that

$$
|\lambda|\left\|\left(\lambda I-A_{\tau}\right)^{-1}\right\|_{L(X)} \leqslant C \quad \text { for } \lambda \in R_{A_{\tau}^{(1)}} \text { with }|\lambda| \geqslant r
$$

where $R_{A_{\tau}^{(1)}}=\left\{\lambda \in \mathbb{C} ; \lambda \neq \omega\left(A_{\tau}^{(1)}\right),\left|\arg \left(\lambda-\omega\left(A_{\tau}^{(1)}\right)\right)\right|<\mu\left(A_{\tau}^{(1)}\right)\right\}$ and $r$ is a sufficiently large positive constant depending on $\bar{\xi}(\tau),\|\bar{v}(\tau, \cdot)\|_{C^{2+\alpha}[0,1]}$.

Proof. First, we prove that $A_{\tau}$ is a sectorial operator in $X$. As stated above, it is known that $A_{\tau}^{(1)}$ is a sectorial operator in $X$ (see [26]). Moreover, according to [21: Section 2.4], if $A_{\tau}^{(2)}$ is a bounded linear operator from $D\left(A_{\tau}^{(1)}\right)$ to $D_{A_{\tau}^{(1)}}\left(\frac{\alpha}{4}, \infty\right)\left(=: X_{\alpha}\right)$ for $\alpha \in(0,1)$, then $A_{\tau}$ is a sectorial operator in $X$. Thus we shall prove

$$
\left\|A_{\tau}^{(2)} U\right\|_{X_{\alpha}} \leqslant C\|U\|_{D\left(A_{\tau}^{(1)}\right)} \quad \text { for } U \in D\left(A_{\tau}^{(1)}\right),
$$

where $C$ is a constant independent of $U$. In fact, one can prove

$$
\left\|\partial_{\eta}^{4} U\right\|_{\infty} \leqslant C_{\tau}\|U\|_{D\left(A_{\tau}^{(1)}\right)},
$$


where $C_{\tau}$ is a constant depending on $\bar{\xi}(\tau),\|\bar{v}(\tau, \cdot)\|_{C^{2+\alpha}[0,1]}$. Then, by means of (A.3) and using this inequality, we get

$$
\begin{aligned}
\left\|A_{\tau}^{(2)} U\right\|_{X_{\alpha}} & \leqslant C_{e q, \tau}\left\|A_{\tau}^{(2)} U\right\|_{C^{\alpha}[0,1]} \\
& =C_{e q, \tau}\left\|C_{1}(\theta)(1-\eta) \frac{\bar{v}_{\eta}(\tau, \cdot)}{\bar{\xi}^{5}(\tau)} \partial_{\eta}^{4} U(0)\right\|_{C^{\alpha}[0,1]} \\
& \leqslant C_{\tau, \theta}\left\|\frac{\bar{v}_{\eta}(\tau, \cdot)}{\bar{\xi}^{5}(\tau)}\right\|_{C^{\alpha}[0,1]} \cdot\left|\partial_{\eta}^{4} U(0)\right| \\
& \leqslant C_{\tau, \theta}^{\prime}\|U\|_{D\left(A_{\tau}^{(1)}\right)^{*}}
\end{aligned}
$$

In particular, we note that a constant $C_{e q, \tau}$ increases with $1 / \bar{\xi}(\tau),\|\bar{v}(\tau, \cdot)\|_{C^{2+\alpha}[0,1]}$. Thus the inequality (A.4) is proved.

Next, we prove the second half of Lemma A.1. For $\lambda \in R_{A_{\tau}^{(1)}}$ and $g \in X$, we consider the resolvent equation

$$
\lambda u-A_{\tau}^{(1)} u-A_{\tau}^{(2)} u=g .
$$

According to [21: Proposition 2.4.1(ii)] again, we obtain that for $|\lambda|$ large enough (A.6) is uniquely solvable with

$$
\|u\|_{D\left(A_{\tau}^{(1)}\right)} \leqslant 2\left(M\left(A_{\tau}^{(1)}\right) \frac{|\lambda|+1}{\left|\lambda-\omega\left(A_{\tau}^{(1)}\right)\right|}+1\right)\|g\| .
$$

On the other hand, from (A.6) we get

$$
|\lambda|\|u\| \leqslant\|u\|_{D\left(A_{\tau}^{(1)}\right)}\left(1+\left\|A_{\tau}^{(2)}\right\|_{L\left(D\left(A_{\tau}^{(1)}\right), X_{\alpha}\right)}\right)+\|g\| .
$$

Using (A.5) and (A.7) in (A.8), we now obtain the desired estimate.

We first prove the inequality (3.14). Let $v$ satisfy (3.10). In order to reduce the inhomogeneous problem (3.10) to a homogeneous problem at the boundaries, we introduce an auxiliary function $\psi$ defined by

$$
\psi(t, \eta):=\left(\eta \bar{\xi}(t) \tan \theta+\frac{\eta^{3}}{3 !} \cdot \frac{3 \tan \theta}{1+\tan ^{2} \theta} \cdot \frac{\bar{v}_{\eta \eta}^{2}(t, 0)}{\bar{\xi}(t)}\right) h(\eta)
$$

where $h \in C^{\infty}[0,1]$ satisfying $h^{\prime}(\eta)<0$ for $\eta \in\left(\frac{1}{4}, \frac{3}{4}\right), h(\eta) \equiv 1$ for $\eta \in\left[0, \frac{1}{4}\right], h(\eta) \equiv 0$ for $\eta \in\left[\frac{3}{4}, 1\right]$. Then it is seen that $v-\psi$ is homogeneous at the boundaries. From this fact and Lemma A.1.(i), we can represent $v-\psi$ as the variation of constants formula by means of the analytic semigroup $\mathrm{e}^{t A_{0}}$. After a simple computation, this formula finally takes the form, for $0 \leqslant t \leqslant T$,

$$
v(t, \cdot)=v^{(1)}(t, \cdot)+v^{(2)}(t, \cdot)+v^{(3)}(t, \cdot)
$$

where

$$
\begin{aligned}
& v^{(1)}(t, \cdot)=\mathrm{e}^{t A_{0}}\left(v_{0}-\psi(0, \cdot)\right), \\
& v^{(2)}(t, \cdot)=\int_{0}^{t} \mathrm{e}^{(t-\sigma) A_{0}}\left[F_{0}(\sigma, \cdot)+\mathcal{A}_{0} \psi(\sigma, \cdot)\right] \mathrm{d} \sigma \\
& v^{(3)}(t, \cdot)=-A_{0} \int_{0}^{t} \mathrm{e}^{(t-\sigma) A_{0}}[\psi(\sigma, \cdot)-\psi(0, \cdot)] \mathrm{d} \sigma+\psi(0, \cdot) .
\end{aligned}
$$


Applying the theory of analytic semigroups (see [21]), we get

$$
\begin{aligned}
& \left\|v^{(1)}\right\|_{\mathcal{Y}^{4+\alpha}\left(\overline{R_{0, T}}\right)} \leqslant C_{0}\left\|v_{0}-\psi(0, \cdot)\right\|_{D_{A_{0}}\left(\frac{2+\alpha}{4}, \infty\right)} \\
& \left\|v^{(2)}\right\|_{\mathcal{Y}^{4+\alpha}\left(\overline{R_{0, T}}\right)} \leqslant C_{0, \alpha} \sup _{0<\delta<T} \delta^{1 / 2} \sup _{t \in[\delta, T]}\left\|F_{0}(t, \cdot)+\mathcal{A}_{0} \psi(t, \cdot)\right\|_{D_{A_{0}}\left(\frac{\alpha}{4}, \infty\right)} .
\end{aligned}
$$

In particular, we can verify that a constant $C_{0}$ increases with $1 / \xi_{0},\left\|v_{0}\right\|_{C^{2+\alpha}[0,1]}$. Hereafter any constant depending on these quantities will be denoted by $C_{0}$.

In order to obtain the estimate of $\left\|v^{(3)}\right\|_{\mathcal{Y}^{4+\alpha}\left(\overline{R_{0, T}}\right)}$, we set

$$
z(t)=\int_{0}^{t} \mathrm{e}^{(t-\sigma) A_{0}}[\psi(\sigma, \cdot)-\psi(0, \cdot)] \mathrm{d} \sigma .
$$

Then $z$ satisfies

$$
v^{(3)}(t, \cdot)=-A_{0} z(t)+\psi(0, \cdot)=-\frac{\mathrm{d}}{\mathrm{d} t} z(t)+\psi(t, \cdot) .
$$

For the function $z$, we have the following estimates.

Lemma A. 2 Let $z$ be a function represented by (A.9). Then, there exist constants $\gamma \in(\alpha, 1)$ and $N=N\left(\xi_{0},\left\|v_{0}\right\|_{C^{2+\alpha}[0,1]}, \alpha, \gamma, K, \theta\right)$ such that

$$
\begin{array}{ll}
\text { (i) } & \left\|\frac{\mathrm{d}}{\mathrm{d} t} z(t)\right\|_{D_{A_{0}}\left(\frac{2+\alpha}{4}, \infty\right)} \leqslant N T^{(\gamma-\alpha) / 4}, \\
\text { (ii) } & \sup _{0<\delta<T} \delta^{1 / 4} \sup _{t \in[\delta, T]}\left\|\frac{\mathrm{d}}{\mathrm{d} t} z(t)\right\|_{D_{A_{0}}\left(\frac{3+\alpha}{4}, \infty\right)} \leqslant N T^{(\gamma-\alpha) / 4}, \\
\text { (iii) } & \sup _{0<\delta<T} \delta^{1 / 2} \sup _{t \in[\delta, T]}\left\|A_{0} \frac{\mathrm{d}}{\mathrm{d} t} z(t)\right\|_{D_{A_{0}}\left(\frac{\alpha}{4}, \infty\right)} \leqslant N T^{(\gamma-\alpha) / 4}, \\
\text { (iv) } & \left\|A_{0} z\right\|_{C^{1 / 2}\left([0, T] ; D_{A_{0}}\left(\frac{\alpha}{4}, \infty\right)\right)} \leqslant N T^{(\gamma-\alpha) / 4} .
\end{array}
$$

Proof. We shall prove the inequality (i). By means of simple calculation, we see that

$$
\begin{aligned}
\frac{\mathrm{d}}{\mathrm{d} t} z(t) & =\int_{0}^{t} A_{0} \mathrm{e}^{(t-\sigma) A_{0}}[\psi(\sigma, \cdot)-\psi(t, \cdot)] \mathrm{d} \sigma+\mathrm{e}^{t A_{0}}[\psi(t, \cdot)-\psi(0, \cdot)] \\
& =: J_{1}(t)+J_{2}(t)
\end{aligned}
$$

Moreover, for $\sigma, t \in[0, T]$, we get

$$
\begin{aligned}
\psi(\sigma, \eta)-\psi(t, \eta)= & \eta h(\eta)(\bar{\xi}(\sigma)-\bar{\xi}(t)) \tan \theta \\
& +\frac{3 \tan \theta}{\left(1+\tan ^{2} \theta\right) \cdot 3 !} \cdot \eta^{3} h(\eta)\left(\frac{\bar{v}_{\eta \eta}^{2}(\sigma, 0)}{\bar{\xi}(\sigma)}-\frac{\bar{v}_{\eta \eta}^{2}(t, 0)}{\bar{\xi}(t)}\right) .
\end{aligned}
$$

First, we shall consider the estimate of $\left\|J_{1}(t)\right\|_{D_{A_{0}}\left(\frac{2+\alpha}{4}, \infty\right)}$. Since $\eta \mapsto \eta h(\eta)$ is in $D_{A_{0}}\left(\frac{\gamma}{4}, \infty\right)$ for any $\gamma \in(0,1)$, we choose $\gamma$ in the interval $(\alpha, 1)$. Then, using the inequalities (A.2) and $\mid \bar{\xi}(t)-$ 
$\bar{\xi}(\sigma) \mid \leqslant 2 K(t-\sigma)^{1 / 2}(\sigma, t \in[0, T], \sigma \neq t)$, we obtain

$$
\begin{aligned}
& \int_{0}^{t}\left\|A_{0} \mathrm{e}^{(t-\sigma) A_{0}}[\eta h(\cdot)(\bar{\xi}(\sigma)-\bar{\xi}(t))]\right\|_{D_{A_{0}}\left(\frac{2+\alpha}{4}, \infty\right)} \mathrm{d} \sigma \\
& \leqslant C_{0} \int_{0}^{t}(t-\sigma)^{-((2+\alpha) / 4-\gamma / 4)-1}\|\eta h\|_{D_{A_{0}}\left(\frac{\gamma}{4}, \infty\right)}|\bar{\xi}(\sigma)-\bar{\xi}(t)| \mathrm{d} \sigma \\
& \leqslant C_{0, K} \int_{0}^{t}(t-\sigma)^{(\gamma-\alpha) / 4-1} \mathrm{~d} \sigma \\
& \leqslant C_{0, K, \alpha, \gamma} T^{(\gamma-\alpha) / 4} .
\end{aligned}
$$

On the other hand, since $\eta \mapsto \eta^{3} h(\eta)$ is in $D_{A_{0}}\left(\frac{2+\gamma}{4}, \infty\right)$ for any $\gamma \in(0,1)$, we are led to

$$
\begin{aligned}
& \int_{0}^{t}\left\|A_{0} \mathrm{e}^{(t-\sigma) A_{0}}\left[\eta^{3} h(\cdot)\left(\frac{\bar{v}_{\eta \eta}^{2}(\sigma, 0)}{\bar{\xi}(\sigma)}-\frac{\bar{v}_{\eta \eta}^{2}(t, 0)}{\bar{\xi}(t)}\right)\right]\right\|_{D_{A_{0}}\left(\frac{2+\alpha}{4}, \infty\right)} \mathrm{d} \sigma \\
& \leqslant C_{0} \int_{0}^{t}(t-\sigma)^{-((2+\alpha) / 4-(2+\gamma) / 4)-1}\left\|\eta^{3} h\right\|_{D_{A_{0}}\left(\frac{2+\gamma}{4}, \infty\right)}\left|\frac{\bar{v}_{\eta \eta}^{2}(\sigma, 0)}{\bar{\xi}(\sigma)}-\frac{\bar{v}_{\eta \eta}^{2}(t, 0)}{\bar{\xi}(t)}\right| \mathrm{d} \sigma \\
& \leqslant C_{0, K} \int_{0}^{t}(t-\sigma)^{(\gamma-\alpha) / 4-1}\left(\left|\bar{v}_{\eta \eta}(\sigma, 0)-\bar{v}_{\eta \eta}(t, 0)\right|+|\bar{\xi}(\sigma)-\bar{\xi}(t)|\right) \mathrm{d} \sigma \\
& \leqslant C_{0, K} \int_{0}^{t}(t-\sigma)^{\gamma / 4-1} \mathrm{~d} \sigma \cdot\left(\left[\bar{v}_{\eta \eta}(\cdot, 0)\right]_{C^{\alpha / 4}[0, T]}+2 K T^{1 / 2-\alpha / 4}\right) \\
& \leqslant C_{0, K, \gamma} T^{\gamma / 4} .
\end{aligned}
$$

Thus we obtain

$$
\left\|J_{1}(t)\right\|_{D_{A_{0}}\left(\frac{2+\alpha}{4}, \infty\right)} \leqslant C_{0, K, \alpha, \gamma, \theta} T^{(\gamma-\alpha) / 4} .
$$

In the same way we also get the estimate of $\left\|J_{2}(t)\right\|_{D_{A_{0}}\left(\frac{2+\alpha}{4}, \infty\right)}$. Hence we obtain the inequality (i).

We can also prove the inequalities (ii), (iii), (iv) in a similar way as above by using the technique in [21: Chapter 4] and leave the details of their proof to the interested reader.

Hence, by means of these estimates, (A.3) and (A.10), we obtain

$$
\left\|v^{(3)}\right\|_{\mathcal{Y}^{4+\alpha}\left(\overline{R_{0, T}}\right)} \leqslant\|\psi(0, \cdot)\|_{C^{2+\alpha}[0,1]}+C_{e q, 0, \alpha, \gamma, K, \theta} T^{(\gamma-\alpha) / 4} .
$$

Consequently, we are led to

$$
\begin{aligned}
\|v\|_{\mathcal{Y}^{4+\alpha}\left(\overline{R_{0, T}}\right) \leqslant} \leqslant & \left\|v^{(1)}\right\|_{\mathcal{Y}^{4+\alpha}\left(\overline{R_{0, T}}\right)}+\left\|v^{(2)}\right\|_{\mathcal{Y}^{4+\alpha}\left(\overline{R_{0, T}}\right)}+\left\|v^{(3)}\right\|_{\mathcal{Y}^{4+\alpha}\left(\overline{R_{0, T}}\right)} \\
\leqslant & C_{0}\left\|v_{0}-\psi(0, \cdot)\right\|_{D_{A_{0}}\left(\frac{2+\alpha}{4}, \infty\right)} \\
& +C_{0, \alpha} \sup _{0<\delta<T} \delta^{1 / 2} \sup _{t \in[\delta, T]}\left\|F_{0}(t, \cdot)+\mathcal{A}_{0} \psi(t, \cdot)\right\|_{D_{A_{0}}\left(\frac{\alpha}{4}, \infty\right)} \\
& +\|\psi(0, \cdot)\|_{C^{2+\alpha}[0,1]}+C_{e q, 0, \alpha, \gamma, K, \theta} T^{(\gamma-\alpha) / 4} .
\end{aligned}
$$

In addition, since $D_{A_{0}}\left(\frac{2+\alpha}{4}, \infty\right)=C_{b_{1}}^{2+\alpha}[0,1]$ and $D_{A_{0}}\left(\frac{\alpha}{4}, \infty\right)=C^{\alpha}[0,1]$ with equivalence of 
norms, we get

$$
\begin{aligned}
\|v\|_{\mathcal{Y}^{4+\alpha}\left(\overline{R_{0, T}}\right)} \leqslant & C_{e q, 0}\left\|v_{0}-\psi(0, \cdot)\right\|_{C^{2+\alpha}[0,1]} \\
& +C_{e q, 0, \alpha} \sup _{0<\delta<T} \delta^{1 / 2} \sup _{t \in[\delta, T]}\left\|F_{0}(t, \cdot)+\mathcal{A}_{0} \psi(t, \cdot)\right\|_{C^{\alpha}[0,1]} \\
& +\|\psi(0, \cdot)\|_{C^{2+\alpha}[0,1]}+C_{e q, 0, \alpha, \gamma, K, \theta} T^{(\gamma-\alpha) / 4} \\
\leqslant & M_{0}+C_{e q, 0, \alpha, K, \theta} T^{\alpha / 4}+C_{e q, 0, \alpha, \gamma, K, \theta} T^{(\gamma-\alpha) / 4}
\end{aligned}
$$

where $M_{0}$ is a constant depending on $\xi_{0},\left\|v_{0}\right\|_{C^{2+\alpha}[0,1]}, \theta$. In particular, we note that $M_{0}$ increases with $1 / \xi_{0},\left\|v_{0}\right\|_{C^{2+\alpha}[0,1]}$. This completes the proof of the inequality (3.14).

Next, we prove the inequality (3.20). Let $V$ satisfy (3.18). In order to obtain a homogeneous problem at the boundaries, we introduce a function $\Psi$ defined by

$$
\Psi(t, \eta)=\left[\eta \bar{\Xi}(t) \tan \theta+\frac{\eta^{3}}{3 !} \cdot \frac{3 \tan \theta}{1+\tan ^{2} \theta} \cdot\left(b_{1}(t) \bar{V}_{\eta \eta}(t, 0)+b_{2}(t) \bar{\Xi}(t)\right)\right] h(\eta) .
$$

Then, since $A_{0}$ is sectorial, we can represent $V$ as

$$
V(t, \cdot)=\int_{0}^{t} \mathrm{e}^{(t-\sigma) A_{0}}\left[\tilde{F}(\sigma, \cdot)+\mathcal{A}_{0} \Psi(\sigma, \cdot)\right] \mathrm{d} \sigma-A_{0} \int_{0}^{t} \mathrm{e}^{(t-\sigma) A_{0}}[\Psi(\sigma, \cdot)-\Psi(0, \cdot)] \mathrm{d} \sigma .
$$

In the same way as we have proved the inequality (3.14), we can also prove the inequality (3.20). So we omit the details. 